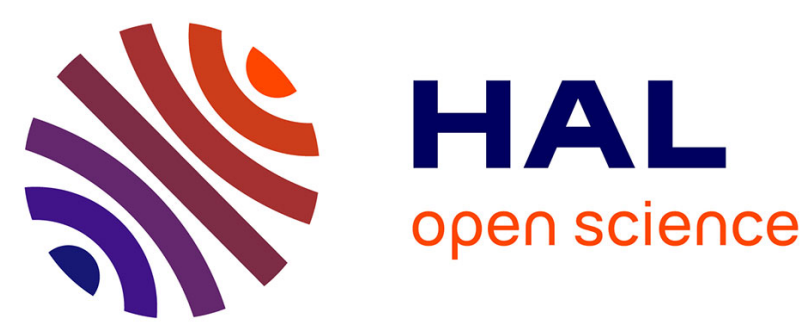

\title{
A nonlinear component mode synthesis method for the computation of steady-state vibrations in non-conservative systems
}

Colas Joannin, Benjamin Chouvion, Fabrice Thouverez, Jean-Philippe Ousty, Moustapha Mbaye

\section{To cite this version:}

Colas Joannin, Benjamin Chouvion, Fabrice Thouverez, Jean-Philippe Ousty, Moustapha Mbaye. A nonlinear component mode synthesis method for the computation of steady-state vibrations in non-conservative systems. Mechanical Systems and Signal Processing, 2016, 83, pp.75 - 92. 10.1016/j.ymssp.2016.05.044 . hal-01389699

\section{HAL Id: hal-01389699 https://hal.science/hal-01389699}

Submitted on 29 Oct 2016

HAL is a multi-disciplinary open access archive for the deposit and dissemination of scientific research documents, whether they are published or not. The documents may come from teaching and research institutions in France or abroad, or from public or private research centers.
L'archive ouverte pluridisciplinaire HAL, est destinée au dépôt et à la diffusion de documents scientifiques de niveau recherche, publiés ou non, émanant des établissements d'enseignement et de recherche français ou étrangers, des laboratoires publics ou privés. 


\title{
A nonlinear component mode synthesis method for the computation of steady-state vibrations in non-conservative systems
}

\author{
Colas Joannin ${ }^{\mathrm{a}, \mathrm{b}, *}$, Benjamin Chouvion ${ }^{\mathrm{a}}$, Fabrice Thouverez $^{\mathrm{a}}$, \\ Jean-Philippe Ousty ${ }^{\mathrm{b}}$, Moustapha Mbaye ${ }^{\mathrm{c}}$ \\ ${ }^{a}$ École Centrale de Lyon, LTDS, CNRS UMR 5513, 69130 Écully, France \\ ${ }^{b}$ Turbomeca, Safran Group, 64511 Bordes, France \\ ${ }^{c}$ Safran-Tech, Safran Group, 91400 Saclay, France
}

\begin{abstract}
This paper presents an extension to classic component mode synthesis methods to compute the steady-state forced response of nonlinear and dissipative structures. The procedure makes use of the nonlinear complex modes of each substructure, computed by means of a modified harmonic balance method, in order to build a reduced-order model easily solved by standard iterative solvers. The proposed method is applied to a mistuned cyclic structure subjected to dry friction forces, and proves particularly suitable for the study of such systems with high modal density and non-conservative nonlinearities.
\end{abstract}

Keywords: reduced-order model, nonlinear mode, component mode synthesis, mistuning, friction

\section{Introduction}

For many years reduced-order modeling of dynamical systems has been a challenging topic in both fundamental and applied sciences, partly due to the rising complexity of modern technologies. The primary objective of order reduction is to cut down the number of parameters describing the system to a much 5 smaller set of variables, while ensuring the capability to predict the behaviour of the initial model with sufficient accuracy. A tremendous diversity of reduced-order modeling techniques applicable to a broad range of engineering problems can be found in the literature. Standard mode superposition, component mode synthesis (CMS) [1, 2, 3, 4], and proper orthogonal decomposition [5] methods, widely used in structural dynamics, are classic examples of model order reduction. The efficiency of these methods mainly relies on the ability 10 of the reduction basis to capture and render the intrinsic dynamics of the system. As a consequence, in the presence of nonlinear phenomena, model reduction can become a very tedious task. Recent works $[6,7]$ have however shown that the concept of nonlinear modes can prove very efficient in building reduced order models of large scale nonlinear systems, thus paving the way for the development of new reduction strategies well-suited for tackling industrial problematics.

Owing to their growing ubiquity in several industrial fields, cyclic structures have been extensively studied by researchers and engineers during the past century, most of the effort aroused by turbomachinary manufacturers in response to a soaring need for efficient power generation for both aeronautical and power plant applications. Facing evermore stringent and conflicting performance and safety requirements, bladed-disk

20 designers have pushed back the limits of these key components to provide industrials with efficient and light structures, while ensuring their integrity in operation. Along with this constant search for an optimal design have emerged new problematics, among which stands out what is commonly referred to as mistuning, which relates to the loss of the cyclic symmetry of the system. A great deal of studies have been dedicated to the understanding and identification of the phenomenon during the last decades, first initiated by Whitehead [8], 25 Wagner [9], Dye and Henry [10], and Ewins [11], and furthered by numerous authors since then. A thorough review is made by Castanier and Pierre in [12], devoted to the state of the art and emerging directions in the

\footnotetext{
${ }^{*}$ Corresponding author

Email address: colas.joannin@doctorant.ec-lyon.fr (Colas Joannin)
} 
modeling and analysis of mistuned bladed-disks.

A practical consequence of mistuning when it comes to modeling is that classic methods taking advantage 30 of the cyclic symmetry property of bladed-disks to reduce the size of the problem [13] cannot be used. Even though basic lumped-parameter models have first allowed to perform various statistical investigations to better understand the phenomenology of such systems $[14,15,16]$, quantitative analyses on large-scale finite element models have required to summon reduced-order modeling techniques, such as the aforementioned classic or modified CMS methods [1, 2, 17], as well as mistuning-dedicated methods [18, 19, 20, 21, 22].

35 However, the introduction of nonlinearities in the model makes most of these techniques either inapplicable or ill-suited to the task, whereas nonlinear effects can yet be of primary importance for the study. As a consequence, the development of new methods allowing to efficiently combine nonlinear phenomena with mistuning has been identified as one of the key challenges in bladed-disk dynamics [12].

So far, the classic way to tackle the mistuning of bladed-disks in a nonlinear context is to summon frequency-based formulation such as harmonic balance methods (HBM), and to proceed to a condensation of the system on the nonlinear degrees of freedom (DOF) [23, 24]. This is conceivable for localised nonlinearities such as contact and friction, and of great interest for the industry. However, for industrial finite element models, the number of nonlinear DOFs easily exceeds a few hundreds per sector, which amounts to 45 thousands overall, resulting in large and cumbersome nonlinear systems, especially when cyclic symmetry cannot or should not be enforced to narrow down the equations to those of one single sector, for instance in case of mistuning or when localised responses are sought. Not to mention the case of non-localised nonlinearities, such as large displacements, for which an exact condensation could amount to keeping all DOFs. As a consequence, the need for fast and reliable design as well as statistical investigations entices both researchers and industrials to endeavour and elaborate new reduction strategies well-suited to such problematics.

In this paper, a new reduced-order modeling technique particularly suitable for the study of nonlinear and mistuned cyclic structures is proposed. The method can be seen as an extension of classic fixed-interface CMS methods, taking advantage of nonlinear complex modes instead of linear modes. This allows to treat 55 internal nonlinear DOFs as slave coordinates, and further the reduction compared to a standard linear CMS. The reduction basis of a superelement is made of nonlinear modes, which must be updated during the computation to properly account for the nonlinear effects, supplemented by a set of static modeshapes through which the substructures are assembled to form the global system. A similar approach was adopted by Apiwattanalunggarn et al. [25] by means of nonlinear normal modes, but is unfortunately limited to weakly 60 nonlinear systems and involves significant analytical developments due to the invariant manifold approach used to compute the modes. The recent and promising works devoted to nonlinear complex modes and their frequency-based formulation, first by Laxalde and Thouverez [6] and later on by Krack et al. [7, 26], have highlighted their capabilities to accurately capture and render the intricate dynamics of strongly nonlinear systems subjected to non-smooth and dissipative nonlinearities. This observation makes of them well-fitted

65 candidates in a model order reduction approach, not to mention the flexibility of their computation, inherent to numerical methods. It should be emphasised that even though the development of the method was initiated to answer topical problematics in bladed-disk dynamics, the procedure could be readily applied to all kind of nonlinear systems, with or without specific symmetry properties.

In section 2, the methodology used to compute nonlinear complex modes, key elements of the method, is reminded. The equations governing a nonlinear superelement are then derived in section 3 . In section 4, the method is applied to the lumped-parameter model of a bladed-disk subjected to blade-disk joint friction, first in the absence of structural mistuning to highlight its capabilities to approximate multi-resonant nonlinear responses, before considering a randomly mistuned system to highlight its effectiveness and efficiency

75 in studying such dynamical systems. The performance and limitations of the method are finally discussed in section 5 . 


\section{Nonlinear complex modes}

The reduced-order modeling technique presented in this paper is inspired by classic CMS, and makes use of the rich information provided by nonlinear complex modes to further the reduction. Key element of the

so procedure, the concept of nonlinear complex modes is first reminded in the following section, along with the main steps of their computation.

\subsection{Historical background}

Nonlinear normal modes of conservative systems have been extensively studied during the last decades. Since Rosenberg adressed the topic in the 1960s [27], many authors have contributed to build a rich and

85 comprehensive literature, thoroughly reviewed by Kerschen et al. in [28]. Vakakis and coworkers used nonlinear normal modes to study the dynamics of cyclic structures subjected to nonlinear restoring forces [29, 30], meanwhile Shaw and Pierre have dedicated their work to build an invariant manifold approach to calculate the modes of weakly nonlinear and damped systems [31]. Later on, Pesheck et al. [32] proposed a modified Galerkin-based approach to extend the invariant manifold method and circumvent some of its limitations. Jézéquel and Lamarque [33] brought new insights through the method of normal forms, and Nayfeh [34] meticulously compared the existing analytical methods to construct nonlinear normal modes of continuous systems. Let us also mention the work by Thomas and Touzé [35, 36], or Arquier et al. [37], dedicated to nonlinear normal modes and internal resonance phenomena. More recently, Peeters et al. [38], and Grolet and Thouverez [39] demonstrated the benefit of numerical techniques, namely shooting methods and HBMs,

95 respectively, to tackle nonlinear normal modes of conservative systems.

In 2009, Laxalde and Thouverez [6] proposed a computation procedure inspired by the HBM to extend the notion of nonlinear modes to a broad class of dissipative systems, capable to handle strong, dissipative and non-smooth nonlinearities. Recent papers [7, 26, 40] have been devoted to these nonlinear complex modes, and proved their efficiency in dealing with a wide range of systems. The nonlinear CMS presented in this paper makes a direct use of these modes, cornerstone of the reduction, and the main steps of their computation is thus reminded hereunder. The interested reader is referred to the aforementioned references for further information.

\subsection{Nonlinear eigenproblem in the frequency domain}

The governing equations of a nonlinear system can usually be written in the following form:

$$
\mathbf{M} \ddot{\mathbf{x}}(t)+\mathbf{C} \dot{\mathbf{x}}(t)+\mathbf{K x}(t)+\mathbf{f}_{n l}(\mathbf{x}, \dot{\mathbf{x}})=\mathbf{f}_{e}(t)
$$

with $\mathbf{M}, \mathbf{C}, \mathbf{K}$ commonly referred to as the mass, viscous damping, and linear stiffness matrices respectively, $\mathbf{f}_{n l}$ a term of nonlinear restoring and dissipative forces, $\mathbf{f}_{e}$ the vector of external load applied to the system, and $\mathbf{x}$ the displacement vector of all DOFs. Overdots indicate time derivatives. In order to take account of the dissipative effects arising from $\mathbf{C}$ and $\mathbf{f}_{n l}$, the solution $\mathbf{x}_{n}$ of the underlying autonomous system (i.e. with $\mathbf{f}_{e}=\mathbf{0}$ ), referred to as nonlinear complex mode, is sought in the form of a damped multi-harmonic oscillation [6]:

$$
\mathbf{x}_{n}(t)=\frac{1}{2}\left\{\sum_{k=1}^{n_{h}} \hat{\mathbf{x}}_{n, k} e^{k t \lambda_{n}}+\text { c.c. }\right\}
$$

with $\hat{\mathbf{x}}_{n, k}$ the $\mathrm{k}$-th harmonic vector of the mode shape, $\lambda_{n}=-\beta_{n}+\mathrm{i} \omega_{n}$ the complex eigenvalue of the mode, and where c.c. denotes the complex conjugate terms. A slightly different definition was later suggested by Krack in [41], which could be used with the method derived in this paper. Equation (2) is then substituted into the autonomous system of Eq. (1), and the residual is orthogonalized with respect to the basis $\left\{e_{k}=e^{\mathrm{i} k t \omega_{n}}\right\}$, $\forall k \in \llbracket 1, n_{h} \rrbracket$, by means of the inner product defined by Eq. (3), where $\boldsymbol{-}$ denotes the complex conjugate.

$$
\langle f \mid g\rangle=\frac{2}{T} \int_{0}^{T} f(t) \overline{g(t)} d t, \text { with } T=\frac{2 \pi}{\omega_{n}}
$$


As fully explained in [6], the decay of the solution is neglected during the period $T$ used in the projection, allowing to make use of the orthogonality of the projection basis. The procedure results in the set of complex algebraic equations given by Eq. (4), coupled through the projection of the nonlinear forces $\left\langle\mathbf{f}_{n l} \mid e_{k}\right\rangle$.

$$
\begin{aligned}
& \forall k \in \llbracket 1, n_{h} \rrbracket: \\
& \mathbf{r}_{k}=\left[\left(k \lambda_{n}\right)^{2} \mathbf{M}+\left(k \lambda_{n}\right) \mathbf{C}+\mathbf{K}\right] \hat{\mathbf{x}}_{n, k}+\left\langle\mathbf{f}_{n l} \mid e_{k}\right\rangle=\mathbf{0}
\end{aligned}
$$

\subsection{Path continuation and phase condition}

The similarity between the previous equations and a standard HBM is obvious. However, it should be pointed out that besides the coefficients $\hat{\mathbf{x}}_{n, k}$, the frequency $\omega_{n}$ and modal damping $\beta_{n}$ are also unknowns. As a consequence, the nonlinear system of Eq. (4) is underdetermined, and requires two additional equations to be solved. In [6], Laxalde and Thouverez suggested to set the amplitude and the phase of the first harmonic of the $\mathrm{j}$-th DOF, $\hat{\mathbf{x}}_{n, 1, j}$, to an arbitrary value $\alpha$. The computation of a mode is thus achieved by performing a sequential continuation on $\alpha$, by enforcing the two conditions of Eq. (5).

$$
\operatorname{Re}\left\{\hat{\mathbf{x}}_{n, 1, j}\right\}=\operatorname{Im}\left\{\hat{\mathbf{x}}_{n, 1, j}\right\}=\alpha
$$

In [40], Joannin et al. suggested to use instead a phase condition, together with an arc-length continuation scheme [42]. Such a continuation procedure requires to supplement the system of Eq. (4) with the following equation:

$$
\sum_{k=1}^{n_{h}}\left\|\delta \hat{\mathbf{x}}_{n, k}\right\|^{2}+\left\|\delta \lambda_{n}\right\|^{2}-d s^{2}=0
$$

where $\delta \hat{\mathbf{x}}_{n, k}$ and $\delta \lambda_{n}$ are the differences between the last converged set of variables and the current trial of the solver, and $\|\bullet\|$ is the canonical norm on $\mathbb{C}^{n}$. The enforcement of Eq. (6) amounts to seeking the solution onto the hypersphere of the configuration space centered on the last converged solution, and of radius $d s$.

Regarding the phase condition, it has been shown that nonlinear modes of cyclic systems can take the form of travelling waves [29, 39, 40], similarly to linear systems [43], and the use of an appropriate phase condition is of the utmost importance in defining the nature of the solution to be computed. To allow the computation of travelling waves, a zero initial velocity for an arbitrary $\mathrm{j}$-th DOF can be used as a phase condition, which is achieved by enforcing Eq. (7) besides Eq. (4) and Eq. (6), where $\hat{\mathbf{x}}_{n, k, j}$ is the j-th component of the k-th harmonic of the nonlinear mode.

$$
\operatorname{Re}\left\{\sum_{k=1}^{n_{h}} k \lambda_{n} \hat{\mathbf{x}}_{n, k, j}\right\}=0
$$

\subsection{Prediction and initialization}

The last step to perform the computation is to provide the iterative solver with a suitable starting point, which can be achieved by means of a variety of predictors [42]. In this work, a secant predictor was implemented, already used in [40]. At the first step of the continuation $i_{0}$, when no previous converged solution 135 is known to use one of these prediction techniques, it has proved good practice to start the continuation for a very low activation of the nonlinearities, and use a linear mode of the equivalent linear system to initialize the nonlinear mode [7, 40]. Thus, prior to the continuation, a standard linear modal analysis of Eq. (4) is performed, after dropping the nonlinear terms $\left\langle\mathbf{f}_{n l} \mid e_{k}\right\rangle$ and applying the relevant transformations to the structural matrices (e.g. bonding nonlinear DOFs), so as to obtain a linear system equivalent to the nonlinear one at low level. Then, the starting point of the solver is taken equal to the linear eigenvector $\hat{\mathbf{x}}_{l}$ pre-multiplied by a small scalar $\epsilon$ to account for the low activation of the nonlinear effects, while the linear eigenvalue $\lambda_{l}$ remains unchanged:

$$
\left(\lambda_{n}, \hat{\mathbf{x}}_{n, 1}, \hat{\mathbf{x}}_{n, 2}, \ldots, \hat{\mathbf{x}}_{n, n_{h}}\right)_{i_{0}}=\left(\lambda_{l}, \epsilon \hat{\mathbf{x}}_{l}, \mathbf{0}, \ldots, \mathbf{0}\right)
$$




\subsection{Numerical aspects}

In practice, an analytical expression for the frequency components of the nonlinear forces, $\left\langle\mathbf{f}_{n l} \mid e_{k}\right\rangle$ in Eq. (4), as a function of the other variables $\hat{\mathbf{x}}_{n, k}$ and $\lambda_{n}$, might not be handy to determine, if ever possible. As an easy and flexible way to get around this difficulty when using frequency-based formulations, a so-called alternating frequency-time (AFT) scheme is implemented here [44]. The method only requires to know the nonlinear law in the time domain, as is usually the case, and uses direct $(\mathcal{F})$ and inverse $\left(\mathcal{F}^{-1}\right)$ Fourier transforms to determine $\left\langle\mathbf{f}_{n l} \mid e_{k}\right\rangle$ at each step of the solver, as summarized in Fig. 1 for a 1-DOF system.

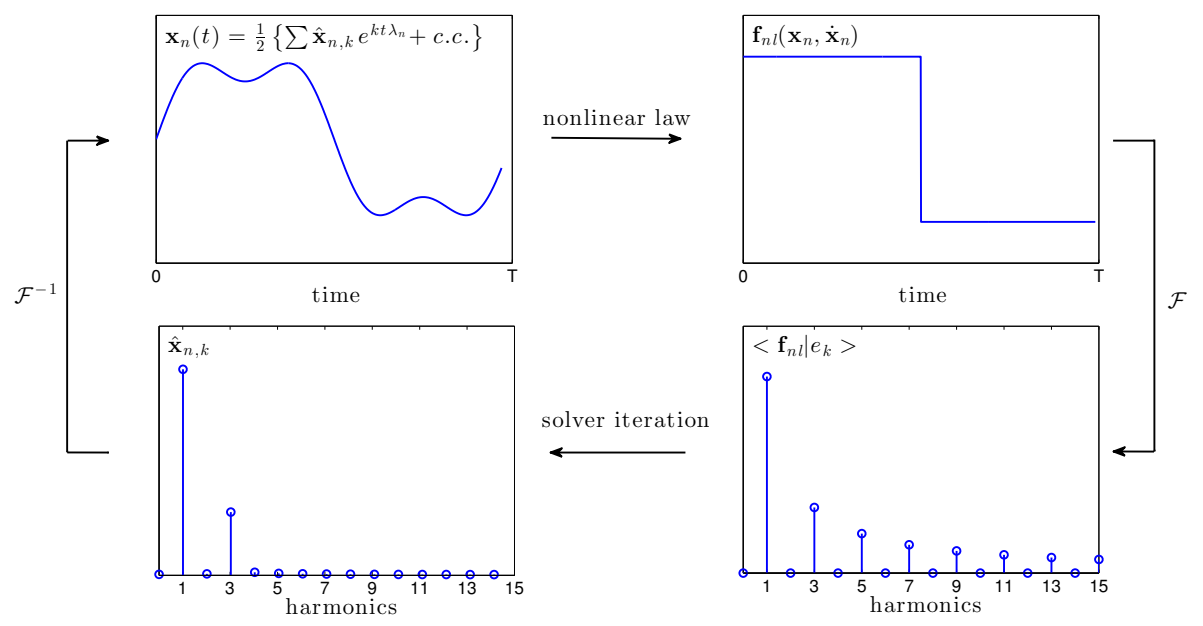

Figure 1: Alternating frequency-time scheme

150

Eventually, the initial set of nonlinear ordinary differential equations of Eq. (1) has been turned into the nonlinear algebraic system of Eq. (4) on the complex variables $\hat{\mathbf{x}}_{n, k}$ and $\lambda_{n}$. Since most numerical solvers only handle real arithmetics, the actual variables of the problem are usually the real and imaginary parts of the unknowns $\left(\hat{\mathbf{x}}_{n, k}^{r}, \hat{\mathbf{x}}_{n, k}^{i}, \omega_{n}\right.$, and $\left.\beta_{n}\right)$, and the set of equations solved is $\forall k \in \llbracket 1, n_{h} \rrbracket:\left[\operatorname{Re}\left(\mathbf{r}_{k}\right) \operatorname{Im}\left(\mathbf{r}_{k}\right)\right]=\mathbf{0}$, where the residual vectors $\mathbf{r}_{k}$ are defined in Eq. (4).

In this study, a trust-region algorithm was used [45] to solve the equations, requiring the knowledge of the Jacobian matrix of the system, i.e. the matrix made of the partial derivatives of the residual vectors with respect to the variables of the problem, given in Eq. (9).

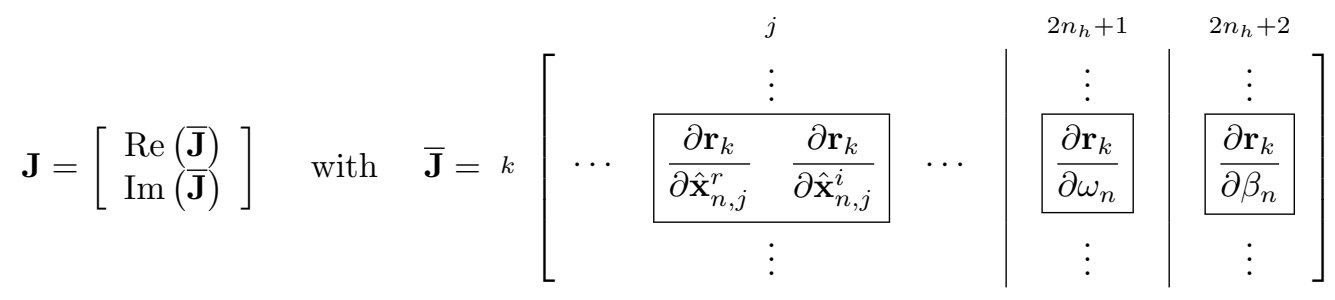

This matrix can be numerically evaluated with a finite difference scheme, but the computational cost might become very significant as the number of variables and the size of the problem increases. A well-known way around this computational burden is to provide the solver with an analytical expression of the partial derivatives, as in Eq. (10), where the chain rule is used to determine the terms resulting from the differentiation 
of $\mathbf{f}_{n l}$.

$$
\left\{\begin{aligned}
\frac{\partial \mathbf{r}_{k}}{\partial \hat{\mathbf{x}}_{n, j}^{r}} & =\left[k^{2} \lambda_{n}^{2} \mathbf{M}+k \lambda_{n} \mathbf{C}+\mathbf{K}\right]+\left(\left\langle\frac{\partial \mathbf{f}_{n l}}{\partial \mathbf{x}_{n}} \mid e_{k}\right\rangle \frac{\partial \mathbf{x}_{n}}{\partial \hat{\mathbf{x}}_{n, j}^{r}}+\left\langle\frac{\partial \mathbf{f}_{n l}}{\partial \dot{\mathbf{x}}_{n}} \mid e_{k}\right\rangle \frac{\partial \dot{\mathbf{x}}_{n}}{\partial \hat{\mathbf{x}}_{n, j}^{r}}\right) \\
\frac{\partial \mathbf{r}_{k}}{\partial \hat{\mathbf{x}}_{n, j}^{i}} & =\left[k^{2} \lambda_{n}^{2} \mathbf{M}+k \lambda_{n} \mathbf{C}+\mathbf{K}\right] \mathrm{i}+\left(\left\langle\frac{\partial \mathbf{f}_{n l}}{\partial \mathbf{x}_{n}} \mid e_{k}\right\rangle \frac{\partial \mathbf{x}_{n}}{\partial \hat{\mathbf{x}}_{n, j}^{i}}+\left\langle\frac{\partial \mathbf{f}_{n l}}{\partial \dot{\mathbf{x}}_{n}} \mid e_{k}\right\rangle \frac{\partial \dot{\mathbf{x}}_{n}}{\partial \hat{\mathbf{x}}_{n, j}^{i}}\right) \\
\frac{\partial \mathbf{r}_{k}}{\partial \omega_{n}} & =\left[2 \mathrm{i} k^{2} \lambda_{n} \mathbf{M}+\mathrm{i} k \mathbf{C}\right] \hat{\mathbf{x}}_{n, k}+\left(\left\langle\frac{\partial \mathbf{f}_{n l}}{\partial \mathbf{x}_{n}} \mid e_{k}\right\rangle \frac{\partial \mathbf{x}_{n}}{\partial \omega_{n}}+\left\langle\frac{\partial \mathbf{f}_{n l}}{\partial \dot{\mathbf{x}}_{n}} \mid e_{k}\right\rangle \frac{\partial \dot{\mathbf{x}}_{n}}{\partial \omega_{n}}\right) \\
\frac{\partial \mathbf{r}_{k}}{\partial \beta_{n}} & =\left[-2 \lambda_{n} k^{2} \mathbf{M}-k \mathbf{C}\right] \hat{\mathbf{x}}_{n, k}+\left(\left\langle\frac{\partial \mathbf{f}_{n l}}{\partial \mathbf{x}_{n}} \mid e_{k}\right\rangle \frac{\partial \mathbf{x}_{n}}{\partial \beta_{n}}+\left\langle\frac{\partial \mathbf{f}_{n l}}{\partial \dot{\mathbf{x}}_{n}} \mid e_{k}\right\rangle \frac{\partial \dot{\mathbf{x}}_{n}}{\partial \beta_{n}}\right)
\end{aligned}\right.
$$

\subsection{Academic example}

In order to illustrate the concept of nonlinear complex modes for a dry friction-damped system, the whole computation procedure presented above is applied to obtain the first nonlinear complex mode of the basic 2-DOFs model of Fig. 2, the parameters of which taking the values reported in Tab. 1. The nonlinear law is not detailed here, but further information are provided in section 4.1.

The so-called backbones of the real and imaginary parts of the complex eigenvalue $\lambda_{n}$, referred to as modal damping $\beta_{n}$ and natural frequency $\omega_{n}$ respectively, are plotted on Fig. 3 as a function of $\left|x_{1}\right|$, amplitude of vibration of the mass $m_{1}$. A frequency shift can be observed due to the softening effect arising from the friction. The natural frequency varies indeed between the corresponding linear natural frequencies of the underlying system with bonded and frictionless interface, at low and high level of $\left|x_{1}\right|$ respectively. The evolution of the modal damping $\beta_{n}$ exhibits a maximum, which accounts for an optimum nonlinear damping arising from the friction element. Similar backbones were already obtained and thoroughly explained in $[6,7,40]$.

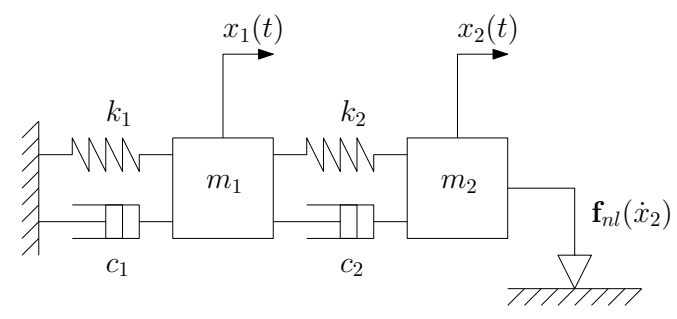

Figure 2: 2-DOFs dry-friction damped model

\begin{tabular}{cccc}
\hline- (unit) & $\mathrm{m}(\mathrm{kg})$ & $\mathrm{c}(\mathrm{Ns} / \mathrm{m})$ & $\mathrm{k}(\mathrm{N} / \mathrm{m})$ \\
\hline DOF1 & 1 & 0.5 & 640 \\
DOF2 & 0.02 & 0.5 & 40 \\
\hline
\end{tabular}

Table 1: Structural parameters of the 2-DOFs system

\section{Nonlinear component mode synthesis}

The governing equations of the new reduced-order modelling technique proposed in this paper are presented in the following sections. The method makes use of nonlinear complex modes to build a reduction basis allowing to significantly reduce the size of the initial nonlinear system, through a substructuring approach. Owing to its similarities with standard CMS methods, this new technique is hereafter referred to as 

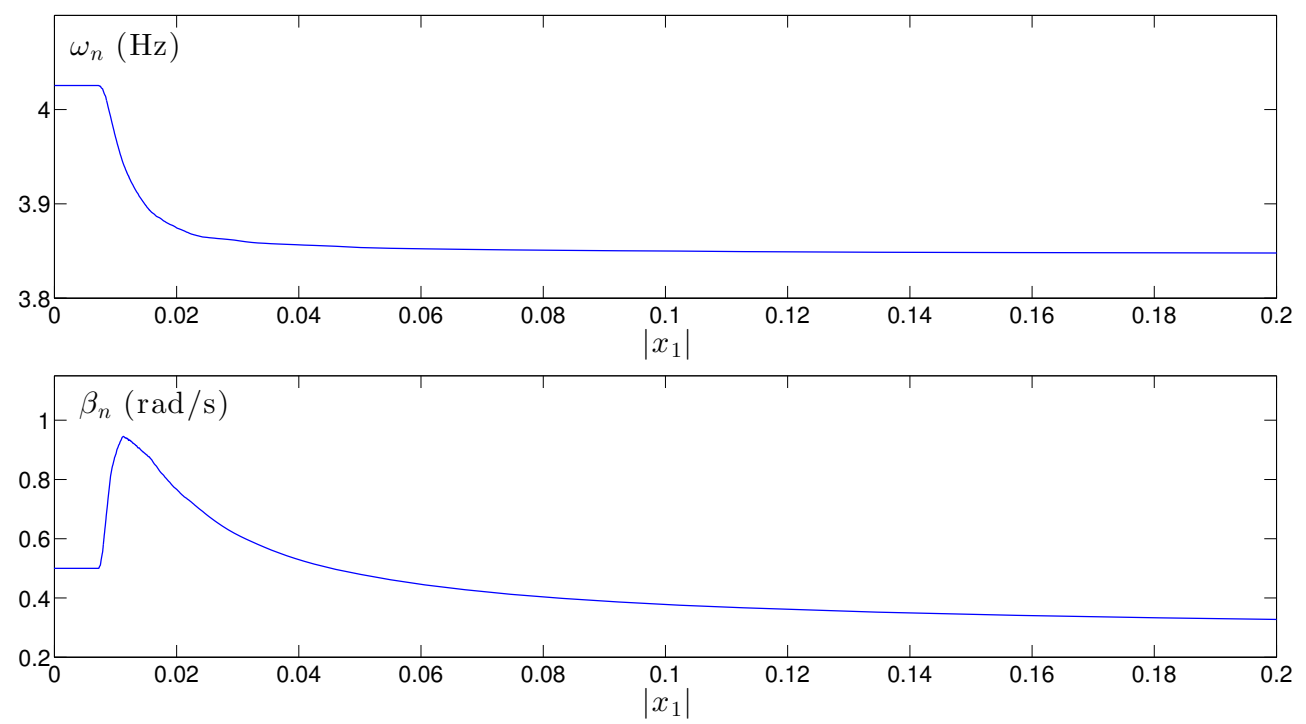

Figure 3: Backbones of the 2-DOFs model

Component Nonlinear Complex Mode Synthesis (CNCMS). The procedure treats independantly each substructure, and can thus be used in combination with other standard CMS methods. The reduction requires the knowledge of at least one nonlinear complex mode, and is focused on determining the steady-state forced response to multi-harmonic excitations of fundamental frequency $\Omega$, in the form given by Eq. (11),

$$
\mathbf{f}_{e}(t)=\frac{1}{2}\left(\sum_{k}\left\langle\mathbf{f}_{e} \mid e_{k}\right\rangle e^{\mathrm{i} k \Omega t}+\text { c.c. }\right)
$$

where c.c. refers to the complex conjugate terms. The analytical expression of a nonlinear superelement is first derived in section 3.1. Then, some numerical aspects of the implementation are tackled in section 3.2.

\subsection{Nonlinear superelements}

As an extension of the classic Craig-Bampton fixed-interface CMS [1] to nonlinear modes, the response of the substructure is approximated by a multi-harmonic sum of nonlinear eigenvectors $\varphi_{n, k}$ computed for fixed boundary conditions, and a set of linear static modeshapes $\boldsymbol{\psi}_{s, k}$ to account for the motion at the interface between two substructures,

$$
\mathbf{x}(t)=\frac{1}{2}\left(\sum_{n} q_{n} \sum_{k} \boldsymbol{\varphi}_{n, k}\left(\left|q_{n}\right|\right) e^{\mathrm{i} k \Omega t}+\sum_{s} p_{s} \sum_{k} \boldsymbol{\psi}_{s, k} e^{\mathrm{i} k \Omega t}+\text { c.c. }\right)
$$

with $q_{n}$ the generalized coordinates of the nonlinear modes, $p_{s}$ the generalized coordinates of the static modes, and where c.c. refers to the complex conjugate terms. The complex eigenvectors $\boldsymbol{\varphi}_{n, k}$ are related to the k-th harmonic coefficient of the $\mathrm{n}$-th mode $\hat{\mathbf{x}}_{n, k}$ of section 2 through the complex generalized coordinates $q_{n}$ according to Eq. (13).

$$
\hat{\mathbf{x}}_{n, k}=q_{n} \boldsymbol{\varphi}_{n, k}
$$


When the DOFs are partitioned into internal and boundary DOFs, the static modeshapes $\boldsymbol{\psi}_{s, k}$ are readily

with,

$$
\begin{aligned}
& \forall k \in \llbracket 1, n_{h} \rrbracket: \\
& \boldsymbol{\psi}_{s, k}=-\mathbf{K}_{i i}^{-1} \mathbf{K}_{i b} \quad \text { with } \quad \mathbf{K}=\left[\begin{array}{cc}
\mathbf{K}_{i i} & \mathbf{K}_{i b} \\
\mathbf{K}_{b i} & \mathbf{K}_{b b}
\end{array}\right]
\end{aligned}
$$

where subscripts $i$ and $b$ refer to internal and boundary DOFs, respectively. Even though the vectors $\boldsymbol{\psi}_{s, k}$ are all equal $\forall k \in \llbracket 1, n_{h} \rrbracket$ here, the method presented in this paper allows to retain different vectors for each $k$ if necessary. It should be stressed that using linear static modeshapes in Eq. (12) is only relevant when the boundaries of the substructure are free from any nonlinear forces, as in section 4 . Replacing these linear vectors by nonlinear static modeshapes is however an interesting prospect of this work.

The modal parameters $\varphi_{n, k}$ and $\lambda_{n}$ of a given nonlinear complex mode are theoretically functions of two independant variables [6]. In Eq. (12), it is however assumed that the amplitude $\left|q_{n}\right|$ of the generalized coordinate suffices to describe the variations of the $n$-th mode. This assumption was already used in $[6,7]$ and provided satisfactory results within a modal synthesis procedure. In the following, the dependency of $\boldsymbol{\varphi}_{n, k}$ and $\lambda_{n}$ over $\left|q_{n}\right|$ is not explicitly written to improve the readability. The substitution of Eq. (12) into the forced system of Eq. (1), followed by a Galerkin projection onto each basis function $\left\{e_{k}=\mathrm{e}^{\mathrm{i} k \Omega t}\right\}$ by means of the inner product defined by Eq. (3) yields,

$$
\begin{aligned}
& \forall k \in \llbracket 1, n_{h} \rrbracket: \\
& {\left[(\mathrm{i} k \Omega)^{2} \mathbf{M}+(\mathrm{i} k \Omega) \mathbf{C}+\mathbf{K}\right]\left\{\sum_{n} q_{n} \boldsymbol{\varphi}_{n, k}+\sum_{s} p_{s} \boldsymbol{\psi}_{s, k}\right\}+\left\langle\mathbf{f}_{n l} \mid e_{k}\right\rangle=\left\langle\mathbf{f}_{e} \mid e_{k}\right\rangle}
\end{aligned}
$$

The projection of the nonlinear force $\left\langle\mathbf{f}_{n l} \mid e_{k}\right\rangle$ is then approximated by the sum of the analogous projections in each eigenvalue problem of Eq. (4) corresponding to the nonlinear modes retained, similarly to what was performed by Krack in [7] for establishing the modal synthesis equation of a single-resonant nonlinear mode. This assumption allows to drop the projection of the nonlinear force in Eq. (15) and substitute instead the opposite of the sum of all the eigenvalue problems, yielding,

$$
\begin{aligned}
& \forall k \in \llbracket 1, n_{h} \rrbracket: \\
& \sum_{n}\left[(\mathrm{i} k \Omega)^{2} \mathbf{M}+(\mathrm{i} k \Omega) \mathbf{C}+\mathbf{K}\right] q_{n} \boldsymbol{\varphi}_{n, k} \\
- & \sum_{n}\left[\left(k \lambda_{n}\right)^{2} \mathbf{M}+\left(k \lambda_{n}\right) \mathbf{C}+\tilde{\mathbf{K}}\right] q_{n} \boldsymbol{\varphi}_{n, k} \\
+ & \sum_{s}\left[(\mathrm{i} k \Omega)^{2} \mathbf{M}+(\mathrm{i} k \Omega) \mathbf{C}+\mathbf{K}\right] p_{s} \boldsymbol{\psi}_{s, k} \\
- & \left\langle\mathbf{f}_{e} \mid e_{k}\right\rangle \approx \mathbf{0}
\end{aligned}
$$

Since the modes were computed for fixed boundary conditions at the interface between two substructures, the stiffness matrix in the eigenvalue problems differs from that of Eq. (15), and is thus denoted $\tilde{\mathbf{K}}$. The nonlinear effects arising from $\left\langle\mathbf{f}_{n l} \mid e_{k}\right\rangle$ in Eq. (15) are now accounted for by the terms on the second line of Eq. (16).

Similarly to a standard Galerkin procedure, the last step of the method is to orthogonalize the set of algebraic equations of Eq. (16) with respect to the family of eigenvectors used to approximate the displacement field, namely $\boldsymbol{\varphi}_{n, k}$ and $\boldsymbol{\psi}_{s, k}$, by means of the standard Hermitian form on $\mathbb{C}^{n}$. It results in the compact set of residual equations given in Eq. (17) governing the displacement of an arbitrary substructure,

$$
\begin{aligned}
& \forall k \in \llbracket 1, n_{h} \rrbracket: \\
& \boldsymbol{\rho}_{k}=\left[\begin{array}{ll}
\boldsymbol{\Phi}_{k}^{\dagger}\left(\mathbf{Z}_{k} \boldsymbol{\Phi}_{k}-\boldsymbol{\xi}_{k}\right) & \boldsymbol{\Phi}_{k}^{\dagger} \mathbf{Z}_{k} \mathbf{\Psi}_{k} \\
\boldsymbol{\Psi}_{k}^{\dagger}\left(\mathbf{Z}_{k} \boldsymbol{\Phi}_{k}-\boldsymbol{\xi}_{k}\right) & \boldsymbol{\Psi}_{k}^{\dagger} \mathbf{Z}_{k} \mathbf{\Psi}_{k}
\end{array}\right]\left\{\begin{array}{c}
\mathbf{q} \\
\mathbf{p}
\end{array}\right\}-\left\{\begin{array}{c}
\boldsymbol{\Phi}_{k}^{\dagger}\left\langle\mathbf{f}_{e} \mid e_{k}\right\rangle \\
\boldsymbol{\Psi}_{k}^{\dagger}\left\langle\mathbf{f}_{e} \mid e_{k}\right\rangle
\end{array}\right\}=\mathbf{0}
\end{aligned}
$$


- $\mathbf{Z}_{k}=\left[(\mathrm{i} k \Omega)^{2} \mathbf{M}+(\mathrm{i} k \Omega) \mathbf{C}+\mathbf{K}\right]$, the linear dynamic stiffness matrix

- $\boldsymbol{\xi}_{k}=\left[\mathbf{M} \boldsymbol{\Phi}_{k} \boldsymbol{\Lambda}^{2} k^{2}+\mathbf{C} \boldsymbol{\Phi}_{k} \boldsymbol{\Lambda} k+\tilde{\mathbf{K}} \boldsymbol{\Phi}_{k}\right]$, the nonlinear matrix resulting from the substitution

- $\boldsymbol{\Phi}_{k}=\left[\cdots \varphi_{n, k} \cdots\right]$, the nonlinear matrix made of the $\mathrm{k}$-th nonlinear eigenvectors

- $\boldsymbol{\Psi}_{k}=\left[\cdots \boldsymbol{\psi}_{s, k} \cdots\right]$, the linear matrix made of the $\mathrm{k}$-th static modeshapes

- $\boldsymbol{\Lambda}=\operatorname{diag}\left[\cdots \lambda_{n} \cdots\right]$, the nonlinear spectral matrix

- $\mathbf{q}=\left[\cdots q_{n} \cdots\right]^{\mathrm{T}}$, the vector of generalized coordinates $q_{n}$

- $\mathbf{p}=\left[\cdots p_{s} \cdots\right]^{\mathrm{T}}$, the vector of generalized coordinates $p_{s}$

and where the superscript $\bullet{ }^{\dagger}$ denotes the Hermitian transpose.

Due to the dependency of the modal properties over the moduli $\left|q_{n}\right|$, an interpolation must be performed at each iteration of the solver to get $\varphi_{n, k}$ and $\lambda_{n}$ for the ongoing value of $q_{n}$. Piecewise linear interpolations have proved good enough to converge when the variations of the modes as a function of $\left|q_{n}\right|$ are known with a relatively good precision.

Eventually, to each substructure of the system correspond $n_{h}$ sets of nonlinear equations such as Eq. (17), which can then all be assembled through the static coordinates $p_{s}$ to build the global reduced-order model. Since more than one harmonic must usually be kept in the series expansion of the mode in Eq. (2) to approximate the nonlinear effects with sufficient accuracy [40], the resulting system of equations is overdetermined. One could choose to retain all the equations and seek an approximate solution by means of dedicated techniques, such as the Levenberg-Marquardt algorithm [46]. However, for an excitation such that $\forall k \neq 1:\left\langle\mathbf{f}_{e} \mid e_{k}\right\rangle=\mathbf{0}$, neglecting the equations corresponding to $k \neq 1$ has proved to yield results in perfect agreement with the solution of the over-determined system. The resulting system is thus square and can be easily solved by standard Newton-like or trust-region algorithms [45].

Neglecting these equations is equivalent neither to assuming mono-harmonic nonlinear modes (i.e. $n_{h}=1$ in Eq. (2)), nor to assuming a mono-harmonic solution in Eq. (12). The terms $\boldsymbol{\Phi}_{1}$ and $\boldsymbol{\Lambda}$ of Eq. (17) are, indeed, always computed by retaining all the harmonics in the system of equations (4), since they can be highly dependent on the harmonic order $n_{h}$, and all the harmonics of the modes are still used to rebuild the multi-harmonic solution from Eq. (12). In section 4, the square formulation is used since the model is subjected to mono-harmonic excitations, with only one nonlinear mode retained per substructure. The flowchart of the whole procedure is provided on Fig. 4.

\subsection{Numerical aspects}

As mentionned in section 2.5, most numerical solvers deal with real arithmetics, and will actually solve $\forall k \in \llbracket 1, n_{h} \rrbracket:\left[\operatorname{Re}\left(\boldsymbol{\rho}_{k}\right) \operatorname{Im}\left(\boldsymbol{\rho}_{k}\right)\right]=\mathbf{0}$ for the real variables $q_{n}^{r}, q_{n}^{i}, p_{s}^{r}$, and $p_{s}^{i}$, real and imaginary parts of $q_{n}$ and $p_{s}$ respectively. Again, since the classic algorithms used to solve nonlinear algebraic systems require the knowledge of the jacobian matrix of the system, a tremendous benefit arises from the knowledge of the analytical expression of the matrix. This expression was derived and provided to the solver, in order to appraise the full potential of the proposed method. The general form of the jacobian matrix is given by:

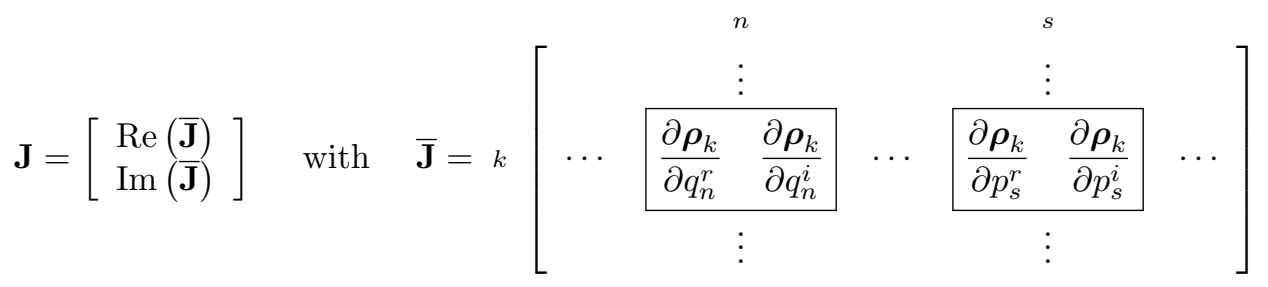




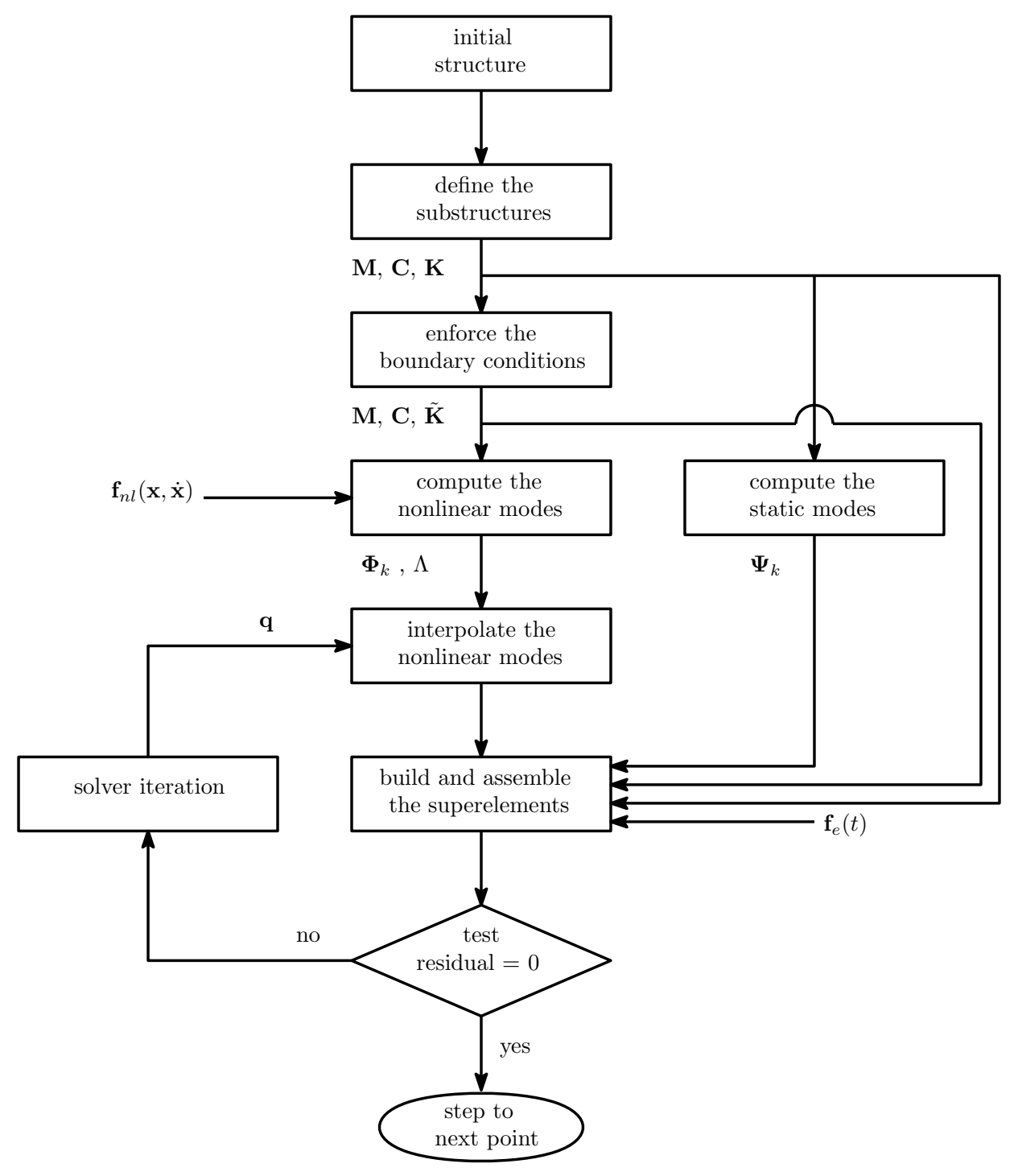

Figure 4: Flowchart of the CNCMS procedure 
The residuals $\boldsymbol{\rho}_{k}$ are written in the form given by Eq. (19) for readability purpose, where all the terms can be easily identified from Eq. (17). The dependency over the moduli $\left|q_{n}\right|$ is explicitly reminded.

$$
\boldsymbol{\rho}_{k}=\left\{\begin{array}{c}
\mathbf{A}\left(\ldots\left|q_{n}\right| \ldots\right) \mathbf{q}+\mathbf{B}\left(\ldots\left|q_{n}\right| \ldots\right) \mathbf{p}-\mathbf{a}\left(\ldots\left|q_{n}\right| \ldots\right) \\
\mathbf{C}\left(\ldots\left|q_{n}\right| \ldots\right) \mathbf{q}+\mathbf{D} \mathbf{p}-\mathbf{b}
\end{array}\right\}
$$

270

with

$$
\begin{aligned}
\mathbf{A}\left(\ldots\left|q_{n}\right| \ldots\right) & =\boldsymbol{\Phi}_{k}^{\dagger}\left(\ldots\left|q_{n}\right| \ldots\right)\left(\mathbf{Z}_{k} \boldsymbol{\Phi}_{k}\left(\ldots\left|q_{n}\right| \ldots\right)-\boldsymbol{\xi}_{k}\left(\ldots\left|q_{n}\right| \ldots\right)\right) \\
\mathbf{B}\left(\ldots\left|q_{n}\right| \ldots\right) & =\boldsymbol{\Phi}_{k}^{\dagger}\left(\ldots\left|q_{n}\right| \ldots\right) \mathbf{Z}_{k} \mathbf{\Psi}_{k} \\
\mathbf{C}\left(\ldots\left|q_{n}\right| \ldots\right) & =\boldsymbol{\Psi}_{k}^{\dagger}\left(\mathbf{Z}_{k} \boldsymbol{\Phi}_{k}\left(\ldots\left|q_{n}\right| \ldots\right)-\boldsymbol{\xi}_{k}\left(\ldots\left|q_{n}\right| \ldots\right)\right) \\
\mathbf{D} & =\boldsymbol{\Psi}_{k}^{\dagger} \mathbf{Z}_{k} \boldsymbol{\Psi}_{k} \\
\mathbf{a}\left(\ldots\left|q_{n}\right| \ldots\right) & =\boldsymbol{\Phi}_{k}^{\dagger}\left(\ldots\left|q_{n}\right| \ldots\right)\left\langle\mathbf{f}_{e} \mid e_{k}\right\rangle \\
\mathbf{b} & =\boldsymbol{\Psi}_{k}^{\dagger}\left\langle\mathbf{f}_{e} \mid e_{k}\right\rangle
\end{aligned}
$$

The expressions of the partial derivatives with respect to $\left|q_{n}\right|$ are,

$$
\begin{aligned}
\frac{\partial \mathbf{A}}{\partial\left|q_{n}\right|} & =\left(\frac{\partial \boldsymbol{\Phi}_{k}}{\partial\left|q_{n}\right|}\right)^{\dagger}\left(\mathbf{Z}_{k} \boldsymbol{\Phi}_{k}-\boldsymbol{\xi}_{k}\right)+\boldsymbol{\Phi}_{k}^{\dagger}\left(\mathbf{Z}_{k} \frac{\partial \boldsymbol{\Phi}_{k}}{\partial\left|q_{n}\right|}-\frac{\partial \boldsymbol{\xi}_{k}}{\partial\left|q_{n}\right|}\right) \\
\frac{\partial \mathbf{B}}{\partial\left|q_{n}\right|} & =\left(\frac{\partial \boldsymbol{\Phi}_{k}}{\partial\left|q_{n}\right|}\right)^{\dagger}\left(\mathbf{Z}_{k} \boldsymbol{\Psi}_{k}\right) \\
\frac{\partial \mathbf{C}}{\partial\left|q_{n}\right|} & =\boldsymbol{\Psi}_{k}^{\dagger}\left(\mathbf{Z}_{k} \frac{\partial \boldsymbol{\Phi}_{k}}{\partial\left|q_{n}\right|}-\frac{\partial \boldsymbol{\xi}_{k}}{\partial\left|q_{n}\right|}\right) \\
\frac{\partial \boldsymbol{\xi}}{\partial\left|q_{n}\right|} & =\mathbf{M}\left(\frac{\partial \boldsymbol{\Phi}_{k}}{\partial\left|q_{n}\right|} \boldsymbol{\Lambda}_{n}^{2}+2 \boldsymbol{\Phi}_{k} \boldsymbol{\Lambda} \frac{\partial \boldsymbol{\Lambda}}{\partial\left|q_{n}\right|}\right)+\mathbf{C}\left(\frac{\partial \boldsymbol{\Phi}_{k}}{\partial\left|q_{n}\right|} \boldsymbol{\Lambda}+\boldsymbol{\Phi}_{k} \frac{\partial \boldsymbol{\Lambda}}{\partial\left|q_{n}\right|}\right)+\tilde{\mathbf{K}} \frac{\partial \boldsymbol{\Phi}_{k}}{\partial\left|q_{n}\right|} \\
\frac{\partial \mathbf{a}}{\partial\left|q_{n}\right|} & =\left(\frac{\partial \boldsymbol{\Phi}_{k}}{\partial\left|q_{n}\right|}\right)^{\dagger}\left\langle\mathbf{f}_{e} \mid e_{k}\right\rangle
\end{aligned}
$$

Using the chain rule to recover the derivatives with respect to the real variables,

$$
\begin{gathered}
\frac{\partial \bullet}{\partial q_{n}^{r}}=\frac{\partial \bullet}{\partial\left|q_{n}\right|} \frac{\partial\left|q_{n}\right|}{\partial q_{n}^{r}}=\frac{\partial \bullet}{\partial\left|q_{n}\right|} \frac{q_{n}^{r}}{\left|q_{n}\right|} \\
\frac{\partial \bullet}{\partial q_{n}^{i}}=\frac{\partial \bullet}{\partial\left|q_{n}\right|} \frac{\partial\left|q_{n}\right|}{\partial q_{n}^{i}}=\frac{\partial \bullet}{\partial\left|q_{n}\right|} \frac{q_{n}^{i}}{\left|q_{n}\right|}
\end{gathered}
$$

and given that

$$
\frac{\partial \mathbf{q}}{\partial q_{n}^{r}}=\{0 \ldots 0,1,0 \ldots 0\}^{\mathrm{T}} \quad, \quad \frac{\partial \mathbf{q}}{\partial q_{n}^{i}}=\{0 \ldots 0, \mathrm{i}, 0 \ldots 0\}^{\mathrm{T}}
$$

the partial derivatives of $\boldsymbol{\rho}_{k}$ with respect to the real variables $q_{n}^{r}$ and $q_{n}^{i}$ are finally obtained,

$$
\frac{\partial \boldsymbol{\rho}_{k}}{\partial q_{n}^{r}}=\left\{\begin{array}{c}
\frac{\partial \mathbf{A}}{\partial q_{n}^{r}} \mathbf{q}+\mathbf{A} \frac{\partial \mathbf{q}}{\partial q_{n}^{r}}+\frac{\partial \mathbf{B}}{\partial q_{n}^{r}} \mathbf{p}-\frac{\partial \mathbf{a}}{\partial q_{n}^{r}} \\
\frac{\partial \mathbf{C}}{\partial q_{n}^{r}} \mathbf{q}+\mathbf{C} \frac{\partial \mathbf{q}}{\partial q_{n}^{r}}
\end{array}\right\} \quad, \quad \frac{\partial \boldsymbol{\rho}_{k}}{\partial q_{n}^{i}}=\left\{\begin{array}{c}
\frac{\partial \mathbf{A}}{\partial q_{n}^{i}} \mathbf{q}+\mathbf{A} \frac{\partial \mathbf{q}}{\partial q_{n}^{i}}+\frac{\partial \mathbf{B}}{\partial q_{n}^{i}} \mathbf{p}-\frac{\partial \mathbf{a}}{\partial q_{n}^{i}} \\
\frac{\partial \mathbf{C}}{\partial q_{n}^{i}} \mathbf{q}+\mathbf{C} \frac{\partial \mathbf{q}}{\partial q_{n}^{i}}
\end{array}\right\}
$$

When a piece-wise linear interpolation of the nonlinear modes is used in the solver, the partial derivatives of $\boldsymbol{\Phi}_{k}$ and $\boldsymbol{\Lambda}$ with respect to $\left|q_{n}\right|$ can be approximated by the slope of the modal components between the two points defining the domain of interpolation. If a higher order of interpolation of the modes is required, the derivatives can then also be approximated by a piece-wise interpolation. Finally, the partial derivatives of $\boldsymbol{\rho}_{k}$ with respect to $p_{s}^{r}$ and $p_{s}^{i}$ are given by,

$$
\frac{\partial \boldsymbol{\rho}_{k}}{\partial p_{s}^{r}}=\left\{\begin{array}{c}
\mathbf{B} \frac{\partial \mathbf{p}}{\partial p_{s}^{r}} \\
\mathbf{D} \frac{\partial \mathbf{p}}{\partial p_{s}^{r}}
\end{array}\right\} \quad \frac{\partial \boldsymbol{\rho}_{k}}{\partial p_{s}^{i}}=\left\{\begin{array}{c}
\mathbf{B} \frac{\partial \mathbf{p}}{\partial p_{s}^{i}} \\
\mathbf{D} \frac{\partial \mathbf{p}}{\partial p_{s}^{i}}
\end{array}\right\}
$$


with

$$
\frac{\partial \mathbf{p}}{\partial p_{s}^{r}}=\{0 \ldots 0,1,0 \ldots 0\}^{\mathrm{T}} \quad \frac{\partial \mathbf{p}}{\partial p_{s}^{i}}=\{0 \ldots 0, \mathrm{i}, 0 \ldots 0\}^{\mathrm{T}}
$$

The matrix J from Eq. (18), corresponding to one superelement, can then be built. The jacobian matrix of the whole system required by the solver is finally obtained by assembling the contributions $\mathbf{J}$ from each superelement.

\section{Numerical applications}

This section provides a few examples to illustrate the capabilities of the CNCMS presented in section 3. In order to test the method, a lumped-parameter model representative of the flexural behaviour of a bladeddisk is first defined in section 4.1. In section 4.2, the response of the perfectly tuned system is then studied for various excitations, and the results are compared to a standard nonlinear modes superposition. In section 4.3, some structural discrepancies are introduced between the sectors, and the procedure is applied to this mistuned model in order to prove its efficiency in tackling such a topical problematic.

\subsection{Lumped-parameter model of bladed disk with dry friction nonlinearities}

The CNCMS procedure derived in section 3 is here applied to a cyclic system consisting of 24 sectors, the fundamental sector being showed on Fig. 5. In the following, the boundary DOFs are the right-hand-side and left-hand-side DOFs of the disk, the interior DOFs referring to the three DOFs of the blade and the central DOF of the disk. The viscous damping coefficients of the dashpots are tuned so as to set the linear damping

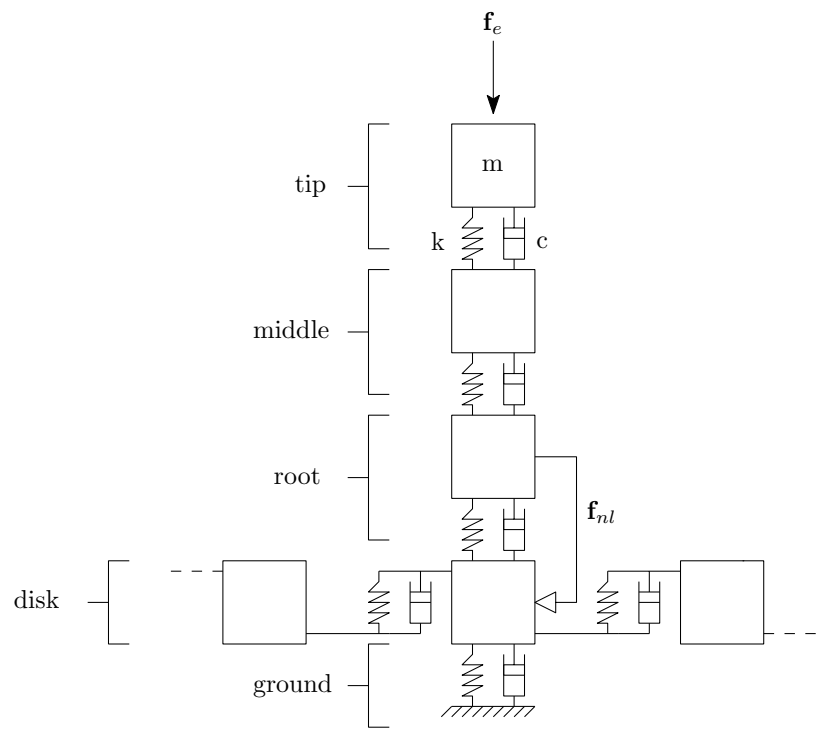

Figure 5: Fundamental sector of the cyclic system

295

ratio of the first mode of the sector to $0.1 \%$. The values taken by the structural parameters, namely mass, viscous damping, and stiffness coefficients, are reported in table 2.

\begin{tabular}{|l|ccccc|}
\hline DOF & 1 (tip) & 2 (middle) & 3 (root) & 4 (disk) & 0 (ground) \\
\hline $\mathrm{m}(\mathrm{kg})$ & 0.2 & 0.3 & 0.4 & 1.2 & - \\
$\mathrm{c}(\mathrm{Ns} / \mathrm{m})$ & 1.3 & 0.7 & 26.7 & 33.3 & 0.4 \\
$\mathrm{k}\left(10^{6} \cdot \mathrm{N} / \mathrm{m}\right)$ & 2 & 1 & 40 & 50 & 0.6 \\
\hline
\end{tabular}

Table 2: Structural parameters of the fundamental sector 
This one-dimensional lumped parameter model was already used by Laxalde [47] and Zhou [48], and aims at being representative of the bending behaviour of an industrial bladed-disk. The evolution of the natural frequencies of the underlying linear system with bonded interface as a function of the nodal diameters is reported on Fig. 6, and exhibits a standard behavior.

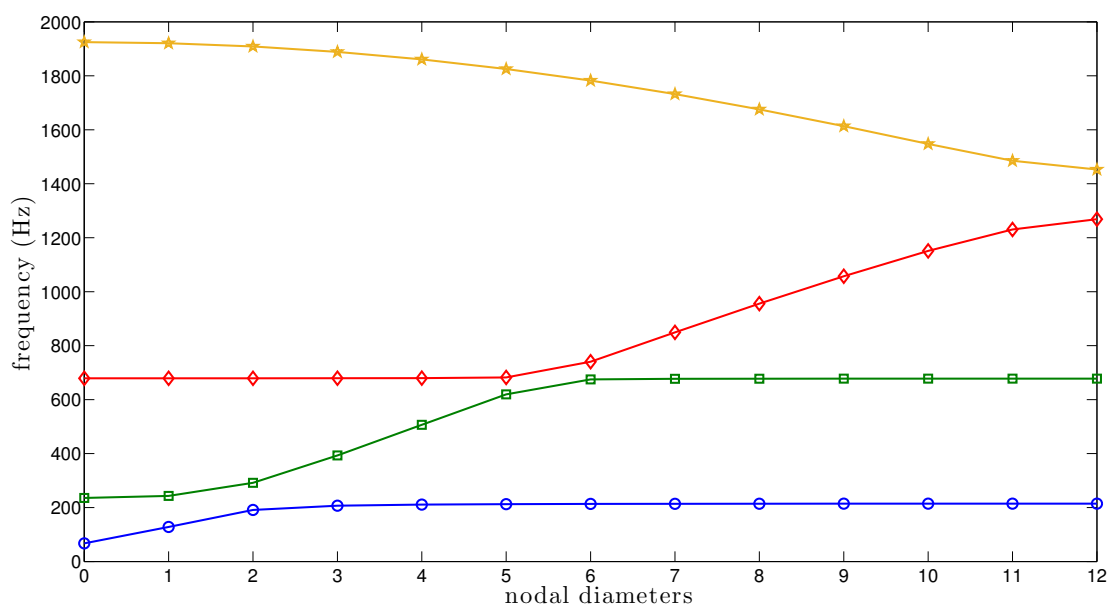

Figure 6: Modal families of the underlying linear system with bonded interfaces (- first, - second, - third, - and fourth family)

The nonlinear nature of the system arises from the dry friction element between the two bottom DOFs on Fig. 5. A regularized version of Coulomb's dry friction law is used, and plotted on Fig. 7. The expression of the jacobian matrix is obtained by standard differentiation, and can be provided to the solver as explained in section 2.5. To compute the vector of nonlinear forces $\mathbf{f}_{n l}$ and the corresponding derivatives of Eq. (10), the following equation is used:

$$
\mathbf{f}_{n l}=\mu N \tanh \left(\frac{\mathbf{P} \dot{\mathbf{x}}_{n}}{\varepsilon}\right) \quad, \quad \frac{\partial \mathbf{f}_{n l}}{\partial \dot{\mathbf{x}}_{n}}=\frac{\mu N}{\varepsilon}\left(1-\left(\tanh \left(\frac{\mathbf{P} \dot{\mathbf{x}}_{n}}{\varepsilon}\right)\right)^{2}\right) \mathbf{P}
$$

with $\mathbf{P}$ the matrix performing the transformation from absolute to relative coordinates for the nonlinear

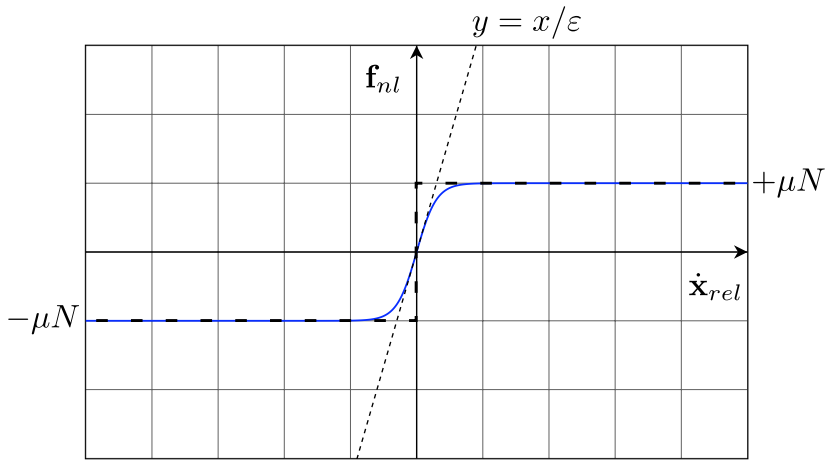

Figure 7: Coulomb's dry friction law (- -) and regularized friction law (-)

DOFs, and setting other components to zero for the linear DOFs. The hyperbolic tangent function in Eq. (27)

is then applied element-wise on the resulting vector to obtain the vector of nonlinear forces $\mathbf{f}_{n l}$. The friction 
coefficient $\mu$ and the normal load at the interface $N$ are set equal to 0.3 and $1000 \mathrm{~N}$, respectively, and the regularization parameter $\varepsilon$ to $0.1 \%$. More sophisticated contact and friction formulations have proved to be perfectly applicable with the nonlinear complex modes computation thanks to the AFT procedure, for instance, taking account of wear or microslip phenomena [49].

315

Travelling wave excitations are ubiquitous in bladed-disk dynamics. They originate from the perturbations in the gas flow arising from the static parts of the turbomachine, which are seen as pressure waves travelling clockwise or anti-clockwise in the frame of reference of the rotating components. As a consequence, in the following subsections, the external load vector $\mathbf{f}_{e}(t)$ of Eq. (1) consists of one or more travelling waves 35

The maximum harmonic order $n_{h}$ in Eq (2) is of the utmost importance in the calculation of the nonlinear complex modes $\left(\omega_{n}, \lambda_{n}\right.$, and $\left.\hat{\mathbf{x}}_{n, k}\right)$, on which relies the accuracy of the solution computed by CNCMS. For this type of systems, it was shown that retaining five harmonics yields satisfactory results [40]. Therefore, in the following computations, the series expansion of Eq. (2) is truncated to $n_{h}=5$.

The first nonlinear complex mode of the fundamental sector with fixed boundary DOFs is computed by means of the methodology presented in section 3, and the two static modeshapes are derived from Eq. (14), allowing to build the superelement from Eq. (17). The backbones of the mode at different levels of nonlinearity are provided on Fig. 8. The evolution of the natural frequency $\omega_{n}$ and modal damping $\beta_{n}$ are similar to Fig. 3, and standard for this type of nonlinearity [6, 7, 40]. A close-up of the modeshape at low and high amplitude of vibration is also provided, so as to point out the change in displacement for the nonlinear DOFs 3 and 4, which are stuck at low level whereas a relative motion can be observed at high level. The two local maxima of the modal damping can be explained by a change in the pattern of relative displacement between DOFs 3 and 4 over a period of vibration, as already observed in [40].
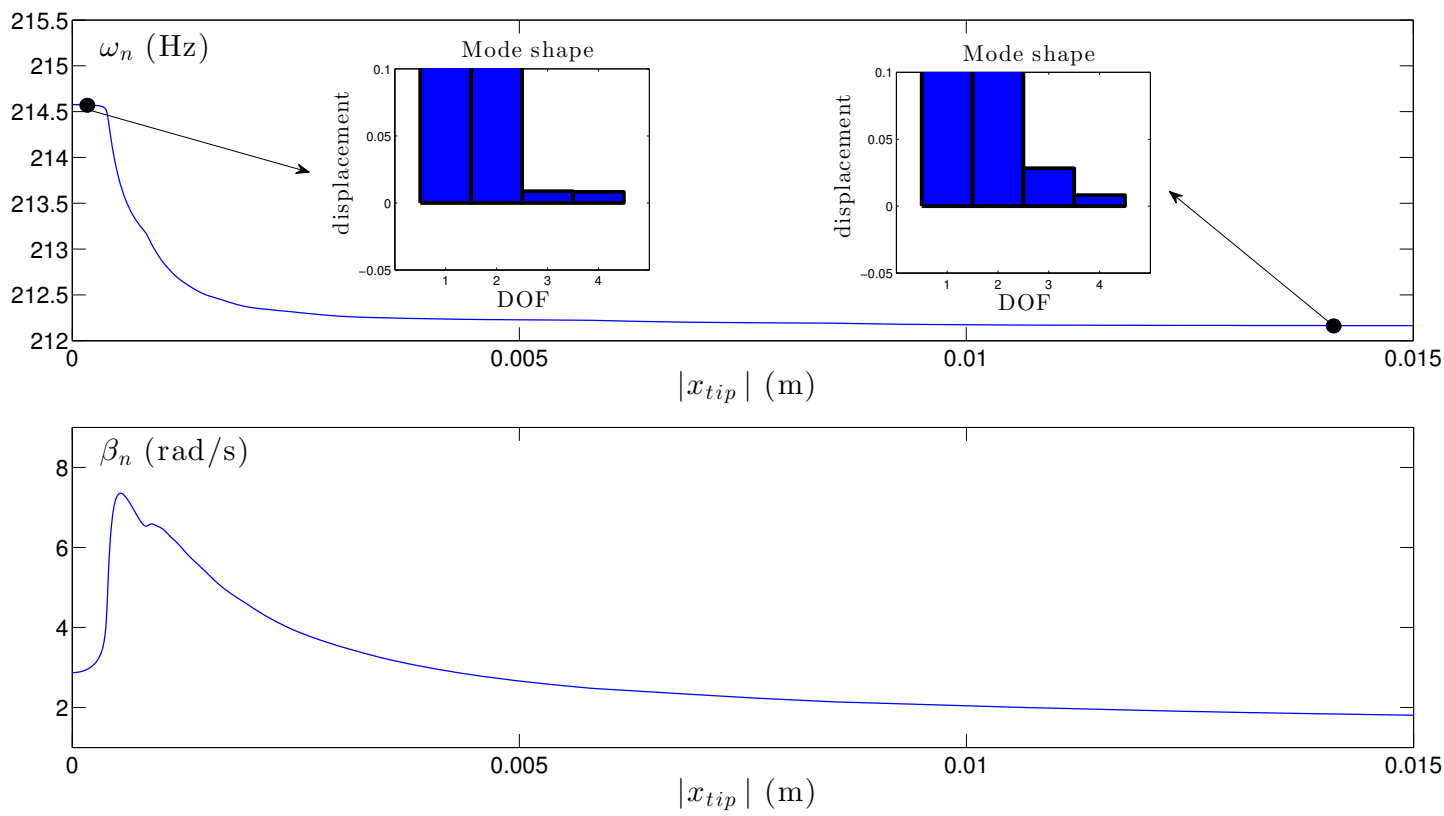

Figure 8: Backbones of the nonlinear complex mode of the fundamental sector applied at the blade-tip of the sectors, as defined by the following equation for the m-th sector:

$$
\mathbf{f}_{e}^{m, n_{d}}(t)=\frac{F}{2}\left(\mathrm{e}^{\mathrm{i}\left(\Omega t+2 \pi m n_{d} / n_{s}\right)}+\text { c.c. }\right) \cdot\{1,0,0,0,0\}^{\mathrm{T}}
$$

where $n_{s}$ is the number of sectors, $n_{d}$ the number of nodal diameters of the travelling wave, $F$ the amplitude of the excitation, and c.c. refers to the complex conjugate term. 


\subsection{Forced response synthesis of the tuned model}

Owing to the perfect cyclic symmetry of the system, the same superelement is used here for all the substructures, built from one fixed-interface nonlinear complex mode and the two static modeshapes of the fundamental sector. A standard travelling wave excitation with 10 nodal diameters is first applied at the blade-tips to generate a high modal contribution from the modes with 10 nodal diameters, and the response is computed for various excitation amplitudes $F \in \llbracket 1,5 \rrbracket N$ in order to sweep the nonlinear domain up to the optimum nonlinear damping point. The response of the blades calculated by CNCMS is plotted on Fig. 9, together with a standard HBM computation to serve as a reference. It can be seen that the proposed method allows in this case to perfectly rebuild the solution.

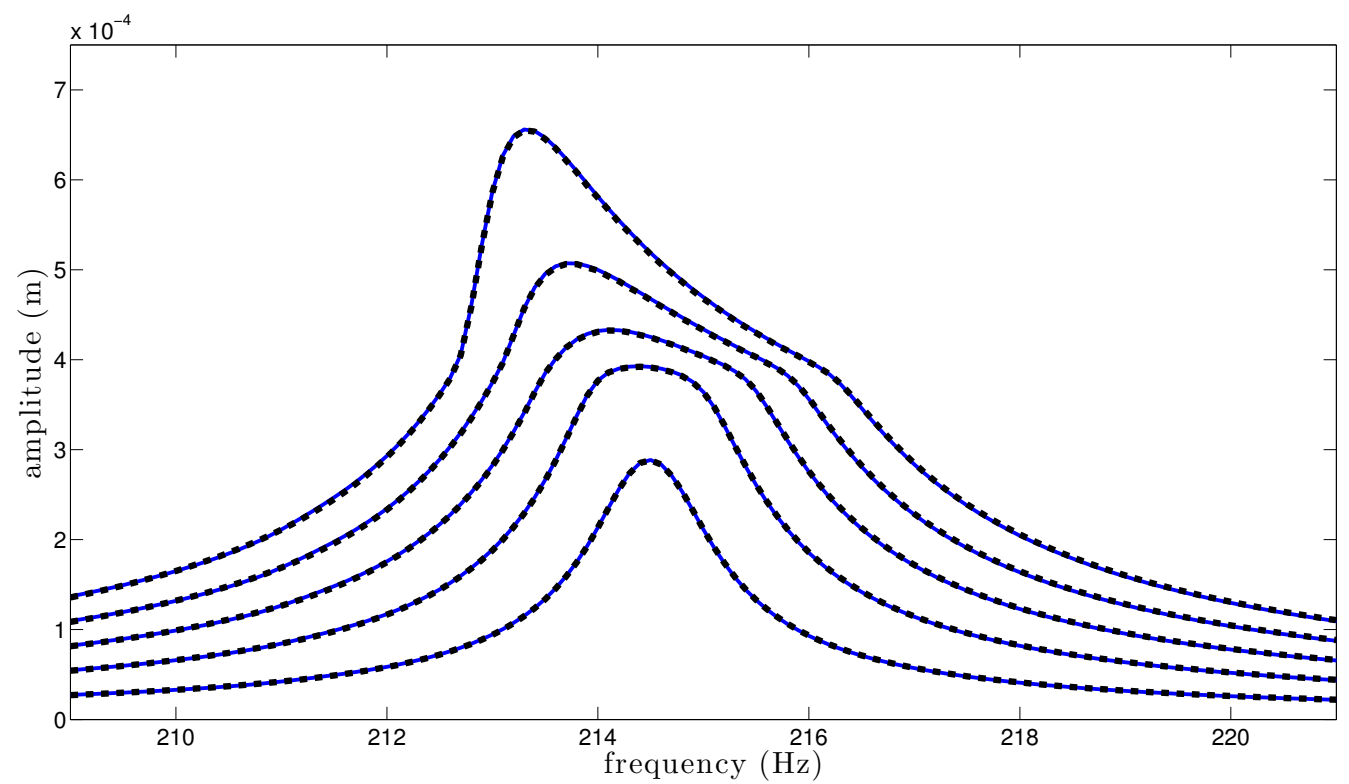

Figure 9: Forced response to a travelling wave excitation with 10 nodal diameters $\forall F \in \llbracket 1,5 \rrbracket N(\mathrm{CNCMS}-$ and HBM - -)

As mentioned in the introduction, not only does the method aim at significantly reducing the number of DOFs retained in the reduced-order model, but it also allows to handle cases of neighboring resonant modes, where standard nonlinear modes superposition is known to fail $[50,51]$. When mode superposition is used to compute the response, the generalized coordinates corresponding to the nonlinear modes are assumed to vary independently of each other, hence the poor accuracy of the method when two close modes have a significant contributions and can interact through the nonlinearities. The equation used to synthesise the forced response by mode superposition is:

$$
\begin{aligned}
\mathbf{x}(t) & =\frac{1}{2}\left(\sum_{n} q_{n} \sum_{k=0}^{n_{h}} \phi_{n, k}\left(\left|q_{n}\right|\right) \mathrm{e}^{\mathrm{i} \Omega t}+\text { c.c. }\right) \\
\text { with } \quad q_{n} & =\frac{\phi_{n, 1}^{\dagger}\left(\left|q_{n}\right|\right) \mathbf{f}_{1}}{\mu_{n}\left(\left|q_{n}\right|\right)\left(\omega_{n}^{2}\left(\left|q_{n}\right|\right)-\Omega^{2}+2 \mathrm{i} \beta_{n}\left(\left|q_{n}\right|\right) \Omega\right)}
\end{aligned}
$$

with $\mathbf{f}_{1}$ the amplitude of the forcing on the whole system, and $\mu_{n}$ the modal mass. It must be understood that in Eq. (29), the modal parameters $\omega_{n}, \beta_{n}$, and $\phi_{n, k}$ are obtained by computing the nonlinear complex modes of the whole system, without substructuring. The interested reader is referred to $[50,51,6,7]$ for further information concerning nonlinear mode synthesis. 
To illustrate the different capabilities between the CNCMS and a standard nonlinear modes superposition, the tuned system is now subjected to an excitation made of the superposition of two travelling waves with different number of nodal diameters, and for different excitation levels, so as to generate simultaneously a significant contribution from the modes exhibiting these diameters. This is performed for 5 and 12 nodal diameters, the corresponding modes of the first family having close natural frequencies (see Fig. 6), and for different excitation levels. The response is plotted on Fig. 10 together with the solutions obtained by CNCMS and HBM. The response obtained by CNCMS appears to provide satisfactory results despite the complexity arising from the two close nonlinear resonances, especially compared to the mode superposition method which dramatically fails at synthesising the response, unable to accurately take account of the simultaneous contribution of the two modes as soon as the system is brought in the nonlinear domain.

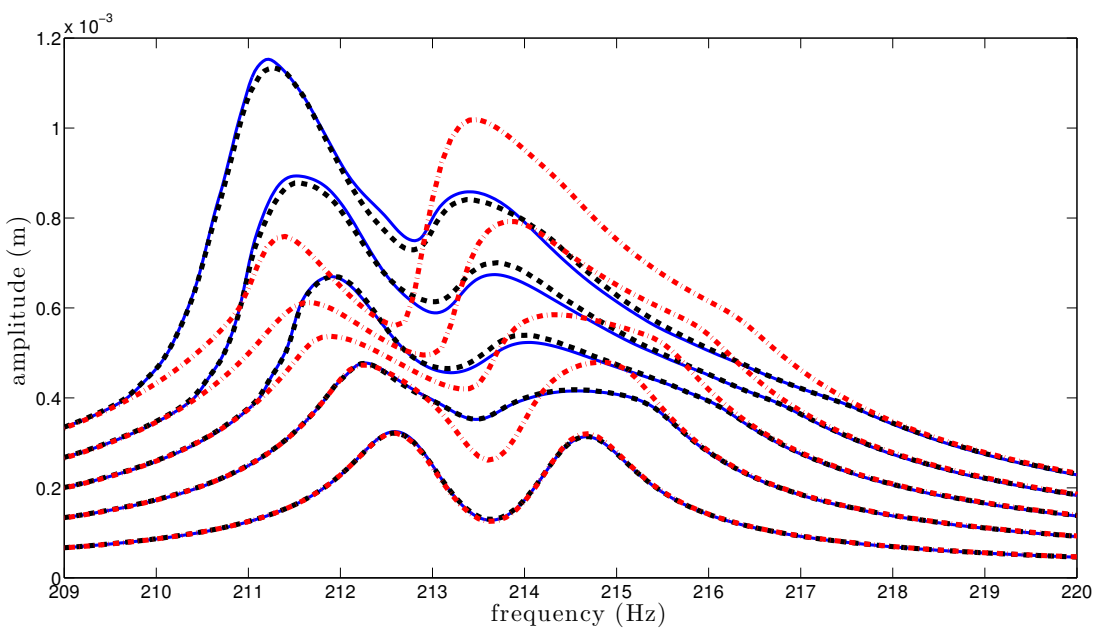

Figure 10: Forced response to a travelling wave excitation with 5 and 12 nodal diameters $\forall F \in \llbracket 1,5 \rrbracket N(\mathrm{CNCMS}-$, HBM - - , and nonlinear modes superposition - - -)

\subsection{Forced response synthesis of the mistuned model}

As already pointed out in the introduction, the development of new reduced-order modeling techniques allowing to efficiently compute the response of mistuned bladed-disks in the presence of nonlinearities is of tremendous importance for both researchers and industrials. The method presented here is devised in order to answer this problematic, and as a consequence its capabilities to deal with such systems must be validated.

In this section, the method is used to synthesise the response of the system defined in section 4.1 when some structural discrepancies break the cyclic symmetry of the structure. A new type of sector is defined, by modifying the tip and middle stiffness coefficients of the fundamental sector 5 , resulting in a $5 \%$ frequency shift of the first mode. The structural parameters of the new sector are reported in table 3 . The fundamental sector of the tuned system (defined in table 2) and this newly defined sector, hereafter referred to as sector $\mathrm{A}$ and sector B, are then randomly distributed around the disk according to the pattern provided in table 4 . The backbones of the first mode of both sectors with clamped boundary conditions are compared on Fig. 11. The $5 \%$ frequency shift mentioned hereabove can be clearly seen on the top plot. The forced response of this mistuned system is then computed by CNCMS and compared to the HBM reference for various excitations.

Figures 12, 13, and 14 show the response of all the blades when subjected to a travelling wave excitation with 6 nodal diameters at low $(F=1 \mathrm{~N})$, medium $(F=5 \mathrm{~N})$, and high $(F=10 \mathrm{~N})$ level of nonlinearity, respectively. Again, the computation is validated with a standard HBM performed on the full system, reported in dashed lines. Even though some small differences can be observed, the results are satisfactory and meet the authors' expectations, especially considering the complexity of the response being synthesised. 


\begin{tabular}{|l|ccccc|}
\hline- (unit) & Tip & Middle & Root & Disk & Ground \\
\hline $\mathrm{m}(\mathrm{kg})$ & 0.2 & 0.3 & 0.4 & 1.2 & - \\
$\mathrm{k}\left(10^{6} \cdot \mathrm{N} / \mathrm{m}\right)$ & 1.8 & 0.9 & 40 & 50 & 0.6 \\
\hline
\end{tabular}

Table 3: Structural parameters of sector B

\begin{tabular}{|l|cccccccccccc|}
\hline Sector & 1 & 2 & 3 & 4 & 5 & 6 & 7 & 8 & 9 & 10 & 11 & 12 \\
\hline Type & A & B & B & A & B & B & B & A & A & A & B & B \\
\hline \hline Sector & 13 & 14 & 15 & 16 & 17 & 18 & 19 & 20 & 21 & 22 & 23 & 24 \\
\hline Type & A & A & B & B & A & A & A & A & A & A & B & B \\
\hline
\end{tabular}

Table 4: Random mistuning pattern
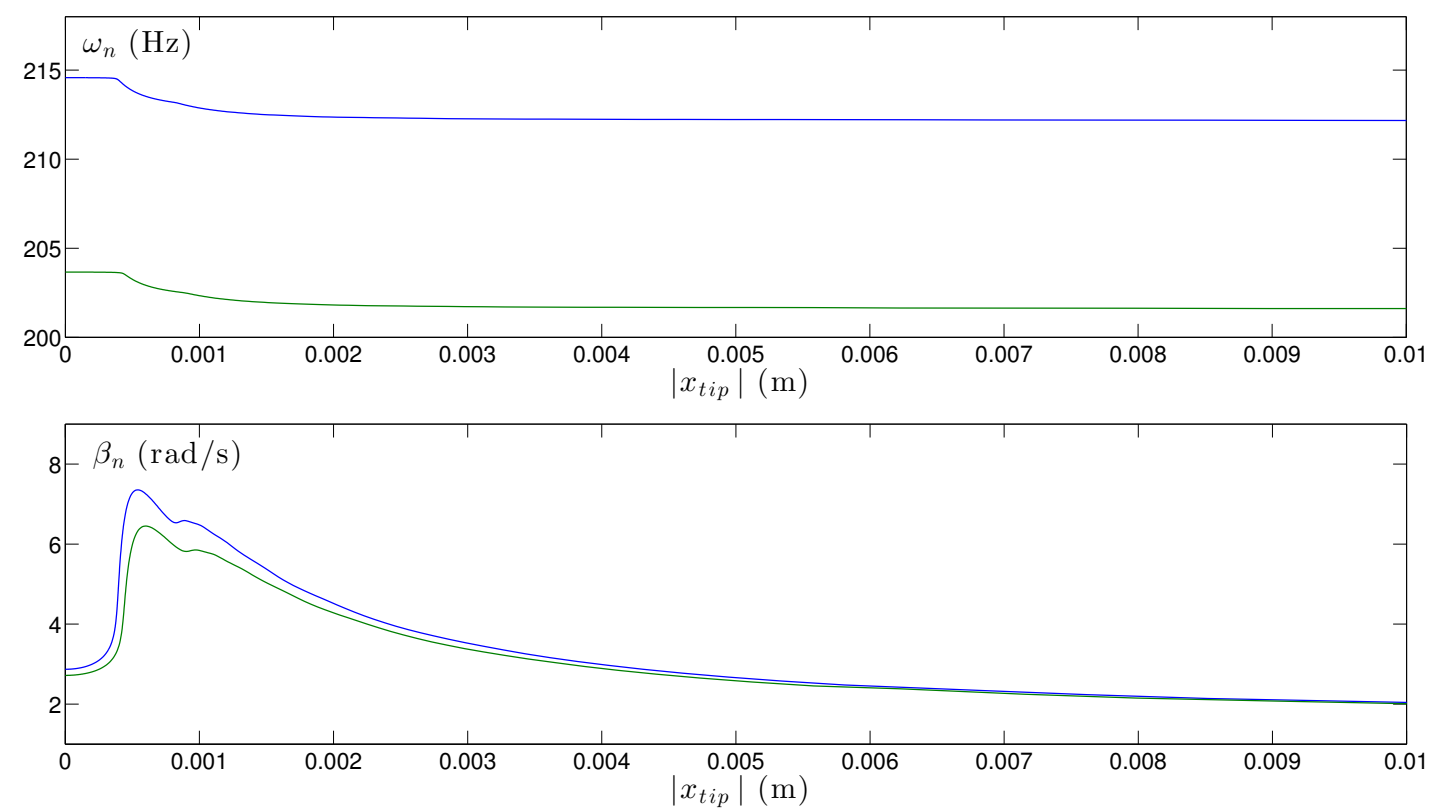

Figure 11: Backbones of the first mode of the two types of sectors with clamped boundary conditions (sector A - and sector B -) 


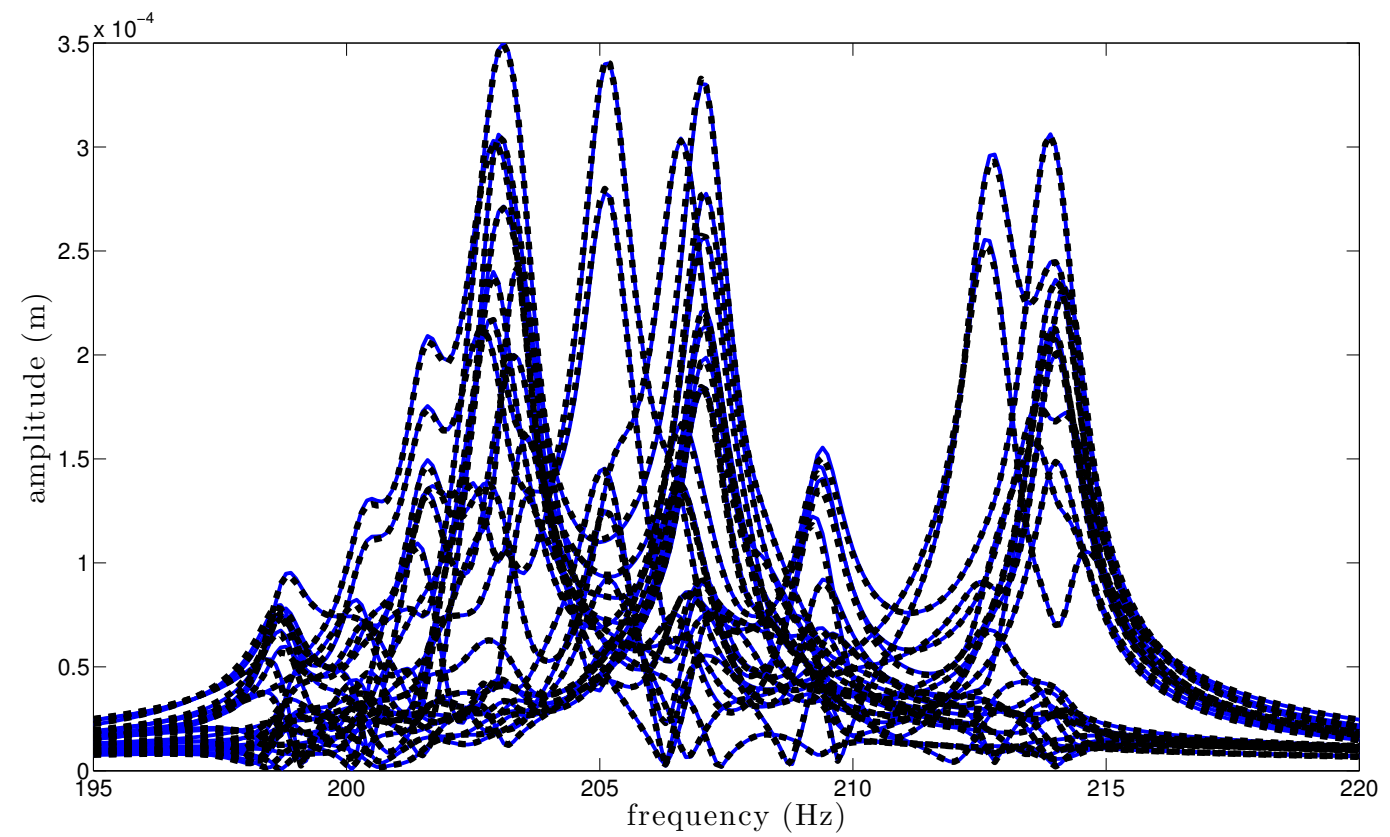

Figure 12: Response of the mistuned system to a travelling wave with 6 nodal diameters and $F=1 \mathrm{~N}(\mathrm{CNCMS}-, \mathrm{HBM}--)$

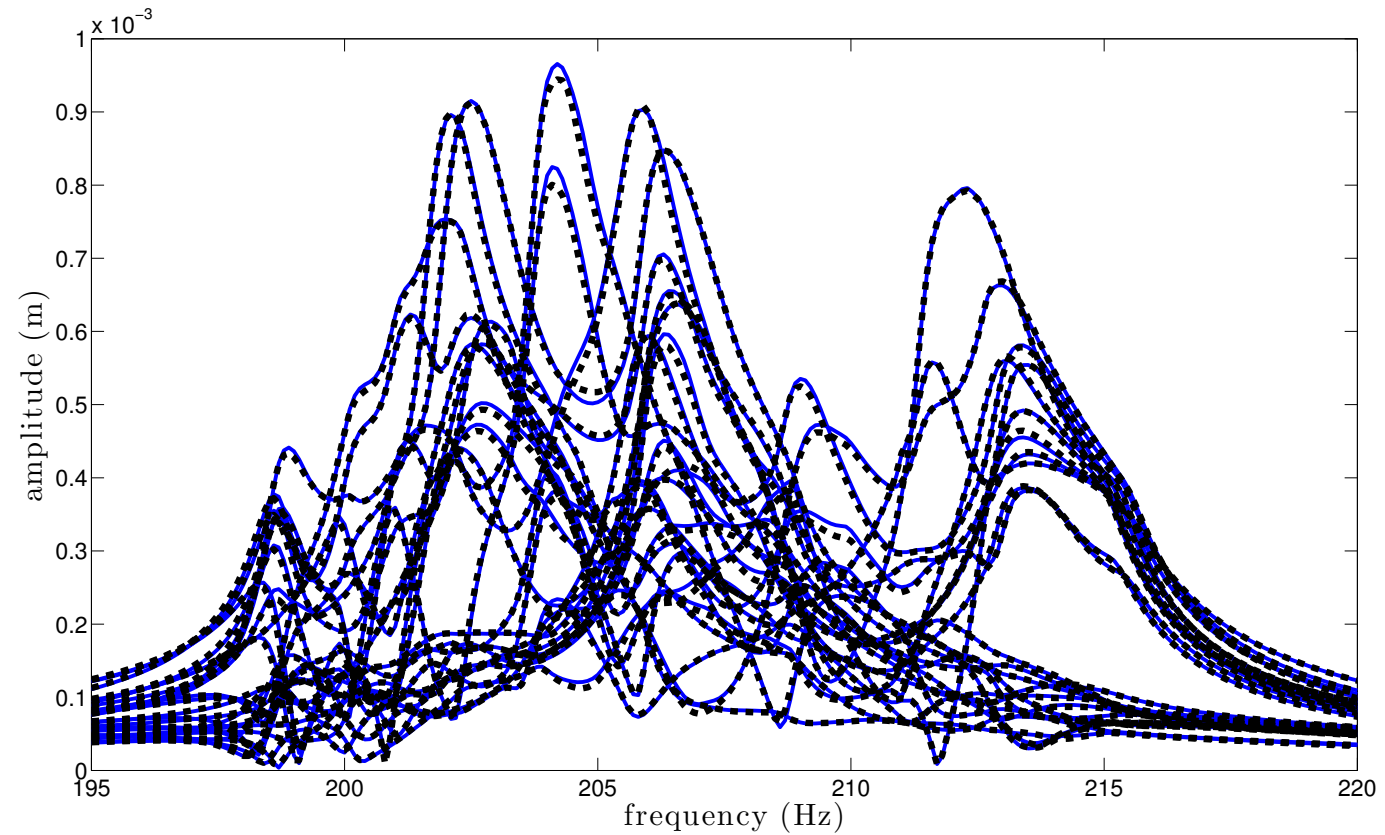

Figure 13: Response of the mistuned system to a travelling wave with 6 nodal diameters and $F=5 \mathrm{~N}(\mathrm{CNCMS}-, \mathrm{HBM}--)$ 


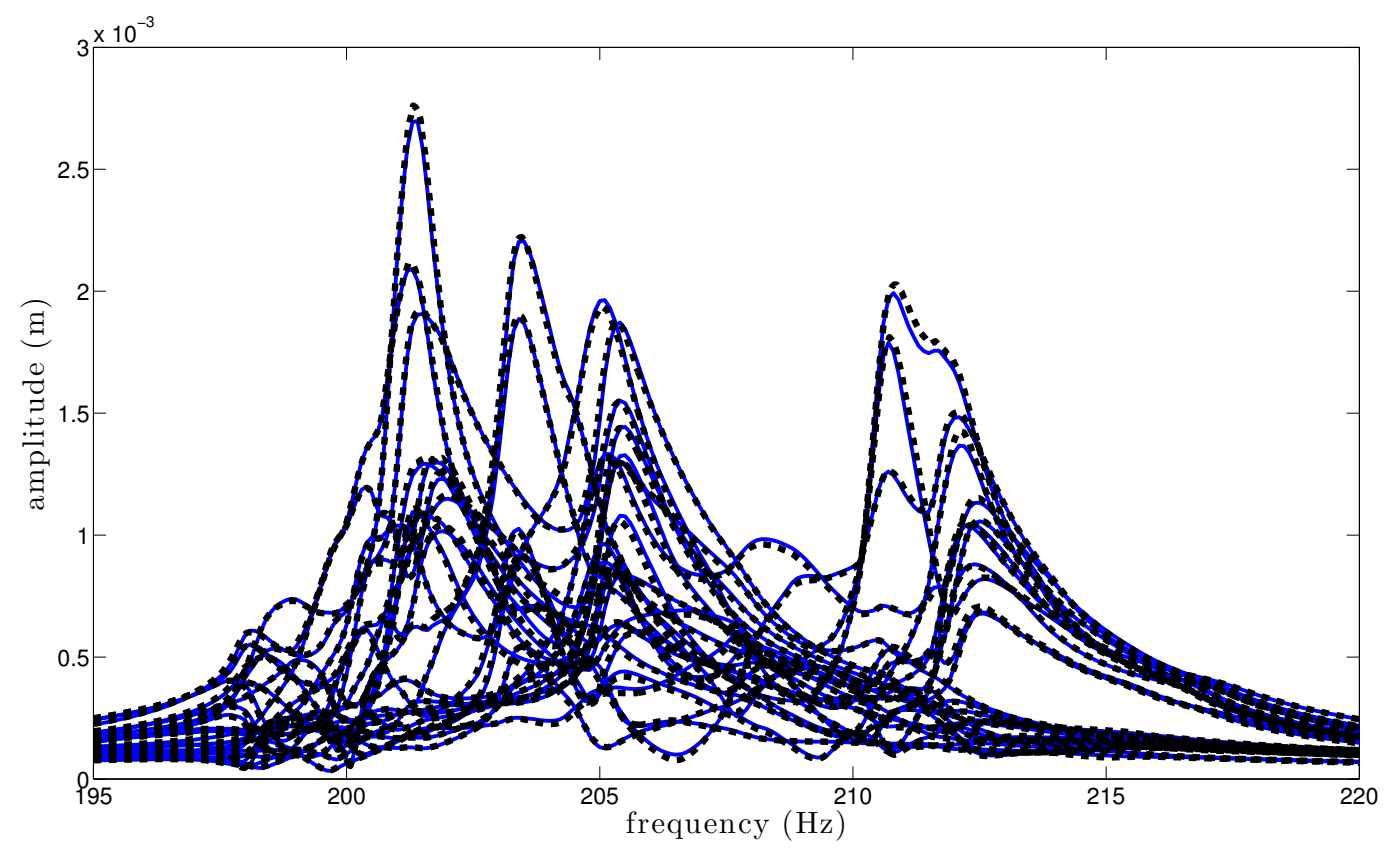

Figure 14: Response of the mistuned system to a travelling wave with 6 nodal diameters and $F=10 \mathrm{~N}(\mathrm{CNCMS}-$, HBM - - )

\section{Discussion}

This section summarizes the performance and limitations of the reduced-order modeling technique derived and tested in the present paper.

\subsection{Computational performance}

In order to evaluate the performance of the proposed method, the evolution of the computation time required to obtain the mistuned responses by HBM, $\forall F \in \llbracket 1,10 \rrbracket N$, is plotted on Fig. 15a, normalized by the computation time required by the CNCMS for comparison purpose. It can be observed that at very low activation of the nonlinearities $(F=1 \mathrm{~N})$, the CNCMS already outperforms the HBM by a ratio of about 25 , which goes up to 77 when the friction is strongly activated $(F=10 \mathrm{~N})$. Near the optimum nonlinear damping point $(F=5 \mathrm{~N})$, the CNCMS is 54 times faster than the HBM. Such a drastic reduction could prove tremendously interesting for large-scale models and statistical investigations, usually involving hundreds or thousands of computations. For information, the order of magnitude of the computational time required for each CNCMS calculation of Fig. 15 is about 10 seconds on a single $3.4 \mathrm{GHz}$ CPU, for all excitation levels.

This first observation highlights the advantage of the proposed method compared to standard HBM procedures, but the benefit could also be pointed out in terms of speed of convergence. Figure 15b shows the evolution of the total number of iterations required by the solver to compute the whole response, as a function of $F$, and exhibits a behaviour similar to the computation time. The number of iterations close to the linear domain is about 2.5 smaller for the CNCMS, and up to 8.5 smaller at very strong nonlinear level, which illustrates the lesser computational effort of the solver.

Even though significant, this decrease in the number of iterations is not the only cause to the overall reduction of the computation time. The performance of the HBM is indeed highly dependent upon the speed of evaluation of the nonlinear forces $\mathbf{f}_{n l}$, whereas for the CNCMS procedure, this term only needs to be evaluated during the computation of the modes, and on a much smaller system than the global structure. As a consequence, a great benefit arises from the CNCMS when a significant part of the computational effort is 
dedicated to the evaluation of the nonlinear forces, for instance in case of intricate, non-smooth nonlinearities such as contact and dry friction.

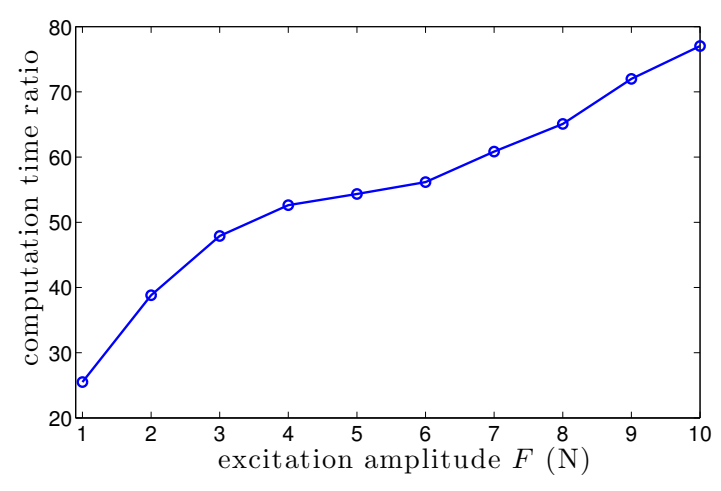

(a) Computation time

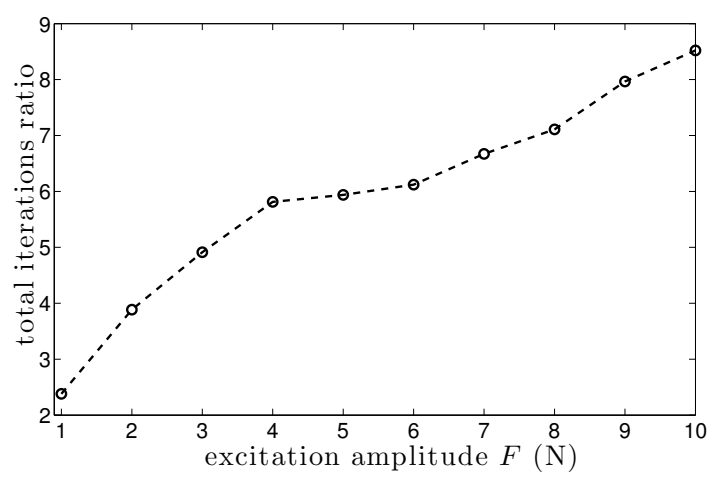

(b) Iterations of the solver

Figure 15: Comparison of the computational effort between the CNCMS and the HBM

The comparison of both methods on Fig.15 does not take into account the computational cost of building the reduced-order model, which can yet be an important parameter. For the CNCMS, this cost amounts to the time required to compute the nonlinear complex modes of the substructures, which takes about 6 seconds on the lumped parameter model of section 4.1. In [6] and [7], Laxalde and Krack have shown that the computation of the nonlinear complex mode of an industrial finite element model, such as a bladed-disk sector with contact and friction nonlinearities, can still be performed at a reasonable cost.

\subsection{Order reduction}

Furthermore, the computational saving pointed out so far should be put in persective with the actual order reduction obtained thanks to the CNCMS. On the lumped-parameter model studied in section 4.1, the procedure results eventually in a relatively small reduction of the overall number of DOFs, owing to the small number of nonlinear DOFs of each substructure. The number of DOFs of the HBM after condensation is equal to 240 , whereas the number of generalized coordinates of the reduced-order model, with one nonlinear complex mode and two static modeshapes per substructure, is equal to 48. As a consequence, the procedure can be expected to be even more efficient on large finite element models comprising many more nonlinear DOFs, compared to standard fixed-interface CMS methods which require to retain all the nonlinear DOFs as master coordinates.

\subsection{Limitations}

This work was initiated in response to the need of efficient reduced-order modeling techniques to tackle the problematic of mistuning of bladed-disks in the presence of nonlinear phenomena. Although the substructuring procedure detailed in the present paper is quite general and applicable to all kind of nonlinear structures,

${ }_{440}$ the method should be expected to be accurate only within a framework verifying the assumptions made in section 3 to derive the expression of the superelements in Eq. (17). In particular, it should be recalled that the motion of the interface between two substructures is approximated by linear static modeshapes, which seems fairly reasonnable when it comes to blade-disk joint friction, but may be less relevant when dealing with some other types of structures. Actually, owing to the dry friction element being directly linked to the boundary DOFs, the lumped-parameter model of section 4.1 does not verify very well this assumption, hence the slight differences observed between the responses computed by CNCMS and the reference when the nonlinearities are strongly activated on Fig. 13 and Fig. 14. The results proved very satisfactory nevertheless, thus highlighting the robustness of the proposed method. 
In section 3.1, the derivation of a nonlinear superelement resulted in a set of coupled equations, equivalent to an over-determined system when the harmonic order $n_{h}$ is larger than 1 . In case of mono-harmonic excitations, it was suggested to neglect the equations corresponding to harmonics different from that of the excitation, so as to deal with a square system. However, in the presence of sub-harmonic or super-harmonic resonances, this assumption could become problematic, because the interactions between harmonics may not be captured by the equations retained. Furthermore, the ability of the nonlinear superelement to account for such phenomena cannot be ensured at this stage, even when the whole over-determined system is solved by means of dedicated algorithms. This would indeed require further investigation, and appears as an interesting prospect of the present work.

\section{Conclusion}

The reduced-order modeling technique derived in this paper, referred to as CNCMS, has been presented and succesfully used to synthesise the forced reponse of a lumped-parameter model of nonlinear bladeddisk. The procedure is quite general and could be used to tackle different kind of structures, but the results obtained here makes it very promising in the study of mistuned bladed-disks subjected to blade-disk joint nonlinearities. The method has proved to significantly outperform standard HBM, especially when nonlinear

465 resonances occur throughout the frequency range of interest, reducing the computation time by a factor of about 50 close to the optimal damping point. The method also proved to provide interesting results in case of neighboring nonlinear modes, where classic nonlinear modes superposition dramatically fails.

In this paper, the CNCMS makes use of fixed-interface nonlinear modes, and is thus the analog of the classic Craig-Bampton CMS [1]. Future prospects include the extension of the method to other CMS techniques, such as free-interface or hybrid CMS [2], or other type of nonlinearities such as large deformation and large displacement. The authors are currently working on the application of the method to a three-dimensional finite element model, in order to appraise its efficiency in dealing with large-scale systems, paving the way for future statistical investigations and robustness analyses in the presence of random and intentional mistuning patterns.

\section{Acknowledgement}

The authors are grateful to Turbomeca - Safran group for providing the financial support allowing to carry out this study.

\section{References}

[1] M. C. C. Bampton, R. R. Craig, Jr., Coupling of substructures for dynamic analyses, AIAA Journal 6 (7) (1968) 1313-1319. doi:10.2514/3.4741.

[2] R. Craig, C. J. Chang, Free-interface methods of substructure coupling for dynamic analysis, AIAA Journal 14 (11) (1976) 1633-1635. doi:10.2514/3.7264.

[3] D. D. Klerk, D. J. Rixen, S. N. Voormeeren, General framework for dynamic substructuring: History, review and classification of techniques, AIAA Journal 46 (5) (2008) 1169-1181. doi:10.2514/1.33274.

[4] K. Bathe, Finite Element Procedures, Prentice Hall, 2006.

[5] G. Kerschen, J.-C. Golinval, A. F. Vakakis, L. A. Bergman, The method of proper orthogonal decomposition for dynamical characterization and order reduction of mechanical systems: An overview, Nonlinear Dynamics 41 (1-3) (2005) 147-169. doi:10.1007/s11071-005-2803-2.

[6] D. Laxalde, F. Thouverez, Complex non-linear modal analysis for mechanical systems: Application to turbomachinery bladings with friction interfaces, Journal of Sound and Vibration 322 (4-5) (2009) 1009-1025. doi:10.1016/j.jsv.2008.11.044. 
[7] M. Krack, L. P. von Scheidt, J. Wallaschek, A method for nonlinear modal analysis and synthesis: Application to harmonically forced and self-excited mechanical systems, Journal of Sound and Vibration 332 (25) (2013) 6798-6814. doi:10.1016/j.jsv.2013.08.009.

[8] D. S. Whitehead, Effect of mistuning on the vibration of turbo-machine blades induced by wakes, Journal of Mechanical Engineering Science 8 (1) (1966) 15-21. doi:10.1243/JMES_JOUR_1966_008_004_02.

[9] J. T. Wagner, Coupling of turbomachine blade vibrations through the rotor, Journal of Engineering for Gas Turbines and Power 89 (4) (1967) 502. doi:10.1115/1.3616718.

[10] R. C. F. Dye, T. A. Henry, Vibration amplitudes of compressor blades resulting from scatter in blade natural frequencies, Journal of Engineering for Power 91 (3) (1969) 182-187. doi:10.1115/1.3574726.

[11] D. Ewins, The effects of detuning upon the forced vibrations of bladed disks, Journal of Sound and Vibration 9 (1) (1969) 65-79. doi:10.1016/0022-460X (69) 90264-8.

[12] M. P. Castanier, C. Pierre, Modeling and analysis of mistuned bladed disk vibration: Current status and emerging directions, Journal of Propulsion and Power 22 (2) (2006) 384-396. doi:10.2514/1.16345.

[13] D. L. Thomas, Dynamics of rotationally periodic structures, International Journal for Numerical Methods in Engineering 14 (1) (1979) 81-102. doi:10.1002/nme.1620140107.

[14] J. H. Griffin, T. M. Hoosac, Model development and statistical investigation of turbine blade mistuning, Journal of Vibration, Acoustics, Stress, and Reliability in Design 106 (2) (1984) 204-210. doi:10.1115/ 1.3269170 .

[15] S.-T. Wei, C. Pierre, Statistical analysis of the forced response of mistuned cyclic assemblies, AIAA Journal 28 (5) (1990) 861-868. doi:10.2514/3.25131.

[16] M. P. Mignolet, W. Hu, Direct prediction of the effects of mistuning on the forced response of bladed disks, Journal of Engineering for Gas Turbines and Power 120 (3) (1998) 626-634. doi:10.1115/1. 2818192.

[17] D.-M. Tran, Component mode synthesis methods using partial interface modes: Application to tuned and mistuned structures with cyclic symmetry, Computers \& Structures 87 (17-18) (2009) 1141-1153. doi:10.1016/j.compstruc. 2009.04.009.

[18] M. P. Castanier, G. Óttarsson, C. Pierre, A reduced order modeling technique for mistuned bladed disks, Journal of Vibration and Acoustics 119 (3) (1997) 439-447. doi:10.1115/1.2889743.

[19] R. Bladh, M. P. Castanier, C. Pierre, Reduced order modeling and vibration analysis of mistuned bladed disk assemblies with shrouds, Journal of Engineering for Gas Turbines and Power 121 (3) (1999) 515-522. doi:10.1115/1.2818503.

[20] M. T. Yang, J. H. Griffin, A reduced-order model of mistuning using a subset of nominal system modes, Journal of Engineering for Gas Turbines and Power 123 (4) (1999) 893-900. doi:10.1115/1.1385197.

[21] A. C. Madden, M. P. Castanier, B. I. Epureanu, Reduced-order model construction procedure for robust mistuning identification of blisks, AIAA Journal 46 (11) (2008) 2890-2898. doi:10.2514/1.37314.

[22] M. Mbaye, C. Soize, J.-P. Ousty, E. Capiez-Lernout, Robust analysis of design in vibration of turbomachines, Journal of Turbomachinery 135 (2) (2012) 021008-021008. doi:10.1115/1.4007442.

[23] S. Nacivet, C. Pierre, F. Thouverez, L. Jézéquel, A dynamic lagrangian frequency-time method for the vibration of dry-friction-damped systems, Journal of Sound and Vibration 265 (1) (2003) 201-219. doi:10.1016/S0022-460X (02)01447-5.

[24] E. P. Petrov, A high-accuracy model reduction for analysis of nonlinear vibrations in structures with contact interfaces, Journal of Engineering for Gas Turbines and Power 133 (10) (2011) 102503-102503. doi:10.1115/1.4002810. 
[25] P. Apiwattanalunggarn, S. Shaw, C. Pierre, Component mode synthesis using nonlinear normal modes, Nonlinear Dynamics 41 (1-3) (2005) 17-46. doi:10.1007/s11071-005-2791-2.

[26] M. Krack, L. P. von Scheidt, J. Wallaschek, On the computation of the slow dynamics of nonlinear modes of mechanical systems, Mechanical Systems and Signal Processing 42 (1-2) (2014) 71-87. doi: $10.1016 / j \cdot y m s s p .2013 .08 .031$.

[27] R. M. Rosenberg, Normal modes of nonlinear dual-mode systems, Journal of Applied Mechanics 27 (2) (1960) 263-268. doi:10.1115/1.3643948.

[28] G. Kerschen, M. Peeters, J. Golinval, A. Vakakis, Nonlinear normal modes, part i: A useful framework for the structural dynamicist, Mechanical Systems and Signal Processing 23 (1) (2009) 170-194. doi: $10.1016 / j$.ymssp. 2008.04.002.

[29] A. F. Vakakis, Dynamics of a nonlinear periodic structure with cyclic symmetry, Acta Mechanica 95 (1-4) (1992) 197-226. doi:10.1007/BF01170813.

[30] A. Vakakis, T. Nayfeh, M. King, A multiple-scales analysis of nonlinear, localized modes in a cyclic periodic system, Journal of Applied Mechanics 60 (2) (1993) 388-397. doi:10.1115/1.2900806.

[31] S. Shaw, C. Pierre, Normal modes for non-linear vibratory systems, Journal of Sound and Vibration 164 (1) (1993) 85 - 124. doi:10.1006/jsvi.1993.1198.

[32] E. Pesheck, C. Pierre, S. W. Shaw, A new galerkin-based approach for accurate non-linear normal modes through invariant manifolds, Journal of Sound and Vibration 249 (5) (2002) 971-993. doi: $10.1006 / j$ svi.2001.3914.

[33] L. Jézéquel, C. H. Lamarque, Analysis of non-linear dynamical systems by the normal form theory, Journal of Sound and Vibration 149 (3) (1991) 429-459. doi:10.1016/0022-460X (91) 90446-Q.

[34] A. H. Nayfeh, On direct methods for constructing nonlinear normal modes of continuous systems, Journal of Vibration and Control 1 (4) (1995) 389-430. doi:10.1177/107754639500100402.

[35] O. Thomas, C. Touzé, A. Chaigne, Non-linear vibrations of free-edge thin spherical shells: modal interaction rules and 1:1:2 internal resonance, International Journal of Solids and Structures 42 (11-12) (2005) 3339-3373. doi:10.1016/j.ijsolstr.2004.10.028.

[36] C. Touzé, O. Thomas, A. Chaigne, Hardening/softening behaviour in non-linear oscillations of structural systems using non-linear normal modes, Journal of Sound and Vibration 273 (1-2) (2004) 77-101. doi:10.1016/j.jsv.2003.04.005.

[37] R. Arquier, S. Bellizzi, R. Bouc, B. Cochelin, Two methods for the computation of nonlinear modes of vibrating systems at large amplitudes, Computers \& Structures 84 (24-25) (2006) 1565-1576. doi: $10.1016 / j$. compstruc. 2006.01.011.

[38] M. Peeters, R. Viguié, G. Sérandour, G. Kerschen, J. C. Golinval, Nonlinear normal modes, part ii: Toward a practical computation using numerical continuation techniques, Mechanical Systems and Signal Processing 23 (1) (2009) 195-216. doi:10.1016/j.ymssp.2008.04.003.

[39] A. Grolet, F. Thouverez, Free and forced vibration analysis of a nonlinear system with cyclic symmetry: Application to a simplified model, Journal of Sound and Vibration 331 (12) (2012) 2911-2928. doi: $10.1016 / j . j s v .2012 .02 .008$.

[40] C. Joannin, B. Chouvion, F. Thouverez, M. Mbaye, J.-P. Ousty, Nonlinear modal analysis of mistuned periodic structures subjected to dry friction, Journal of Engineering for Gas Turbines and Power 138 (7) (2015) 072504-072504. doi:10.1115/1.4031886.

[41] M. Krack, Nonlinear modal analysis of nonconservative systems: Extension of the periodic motion concept, Computers and Structures 154 (2015) 59-71. doi:10.1016/j.compstruc.2015.03.008. 
[42] A. H. Nayfeh, B. Balachandran, Applied Nonlinear Dynamics, Wiley-VCH Verlag GmbH, 1995, Ch. 6, pp. 423-460. doi:10.1002/9783527617548.ch6.

[43] D. J. Ewins, Vibration characteristics of bladed disc assemblies, Journal of Mechanical Engineering Science 15 (3) (1973) 165-186. doi:10.1243/JMES_JOUR_1973_015_032_02.

[44] T. M. Cameron, J. H. Griffin, An alternating frequency/time domain method for calculating the steadystate response of nonlinear dynamic systems, Journal of Applied Mechanics 56 (1) (1989) 149-154. doi:10.1115/1.3176036.

[45] A. Conn, N. Gould, P. Toint, Trust Region Methods, Society for Industrial and Applied Mathematics, 2000. doi:10.1137/1.9780898719857.

[46] G. A. Watson, J. J. Moré, Lecture Notes in Mathematics - The Levenberg-Marquardt algorithm: Implementation and theory, Vol. 630, Springer Berlin Heidelberg, 1978. doi:10.1007/BFb0067700.

[47] D. Laxalde, F. Thouverez, J.-J. Sinou, J.-P. Lombard, Qualitative analysis of forced response of blisks with friction ring dampers, European Journal of Mechanics - A/Solids 26 (4) (2007) 676-687. doi: $10.1016 / j$. euromechsol.2006.10.002.

[48] B. Zhou, F. Thouverez, D. Lenoir, Vibration reduction of mistuned bladed disks by passive piezoelectric shunt damping techniques, AIAA Journal 52 (6) (2014) 1194-1206. doi:10.2514/1. J052202.

[49] D. Laxalde, L. Salles, L. Blanc, F. Thouverez, Non-linear modal analysis for bladed disks with friction contact interfaces, in: ASME Turbo Expo 2008: Power for Land, Sea, and Air, Vol. 5, 2008, pp. 457-467. doi:10.1115/GT2008-50860.

[50] W. Szemplińska-Stupnicka, The modified single mode method in the investigations of the resonant vibrations of non-linear systems, Journal of Sound and Vibration 63 (4) (1979) 475-489. doi:10.1016/ 0022-460X (79) 90823-X.

[51] C. Gibert, Fitting measured frequency response using non-linear modes, Mechanical Systems and Signal Processing 17 (1) (2003) 211-218. doi:10.1006/mssp.2002.1562. 